\title{
Stability of Certainty and Opinion on Influence Networks
}

by

\author{
Ariel Webster \\ B.A., St. Mary's College of Maryland, 2012 \\ A Thesis Submitted in Partial Fulfillment of the \\ Requirements for the Degree of \\ Master of Science \\ in the Department of Computer Science
}

(C) Graduate Advisor, 2016

University of Victoria

All rights reserved. This thesis may not be reproduced in whole or in part, by photocopying or other means, without the permission of the author. 
Stability of Certainty and Opinion on Influence Networks

by

Ariel Webster

B.A., St. Mary's College of Maryland, 2012

Supervisory Committee

Dr. Bruce Kapron, Supervisor

(Department of Computer Science)

Dr. Valerie King, Supervisor

(Department of Computer Science) 


\title{
Supervisory Committee
}

Dr. Bruce Kapron, Supervisor

(Department of Computer Science)

Dr. Valerie King, Supervisor

(Department of Computer Science)

\begin{abstract}
This thesis introduces a new model to the field of opinion dynamics in which each node in a network moves to the mass center of the opinions in its neighborhood weighted by the changing certainty each node has in its own opinion. An upper bound of $O(n)$ is proved for the number of timesteps until this model reaches a stable state. A second model is also analyzed in which nodes move to the mass center of the opinions of the nodes in their neighborhood unweighted by the certainty those nodes have in their opinions. This second model is shown to have a $O(d)$ time complexity on a tree, where $d$ is the diameter of the network, and is compared with a very similar model presented in 2013 by Frischknecht, Keller, and Wattenhofer [22] who found a lower bound on some networks of $\Omega\left(\frac{3}{2}\right)$.
\end{abstract}




\section{Contents}

Supervisory Committee ii

Abstract $\quad$ iii

Table of Contents $\quad$ iv

List of Figures $\quad$ vi

1 Introduction 1

2 Background $\quad 3$

2.1 Opinion Dynamics . . . . . . . . . . . . . . . . . 3

2.1.1 Influence . . . . . . . . . . . . . . . 3

2.1.2 Conformity, Compromise, and Stubbornness . . . . . . . . 5

2.2 Information Cascading . . . . . . . . . . . . . 6

3 The Model $\quad 7$

3.1 The Models . . . . . . . . . . . . . . . . . . . . . . 7

3.2 The Networks . . . . . . . . . . . . . . . . . . . . . . 10

4 Analysis of Certainty Model $\quad 11$

4.1 Tests of Model . . . . . . . . . . . . . . . . . . . . 11

4.2 Stability . . . . . . . . . . . . . . . . . 13

4.3 Uniformity and Standoff . . . . . . . . . . . . . . . 16

4.4 Stable Uniformity . . . . . . . . . . . . . . . . . . . 18

4.4.1 Time from Uniformity to Stability . . . . . . . . . . . . . . . . 18

4.4.2 Time until Uniformity is Reached . . . . . . . . . . . . . . 22

4.5 Certainty of a Uniform Stable Graph . . . . . . . . . . . . . . . 25

4.6 Stable Standoff . . . . . . . . . . . . . . . . 26

4.7 Stability of Number . . . . . . . . . . . . . . . 28 
5 Analysis of Pure Opinion Model 32

5.1 Trees ................................ 34

5.2 A $\Omega\left(n^{\frac{3}{2}}\right)$ Lower Bound . . . . . . . . . . . . . . . . 35

$\begin{array}{lll}6 & \text { Future Work } & 47\end{array}$

$\begin{array}{ll}\text { Bibliography } & 49\end{array}$ 


\section{List of Figures}

Figure 4.1 Mean Timesteps to Stability on Certainty Model . . . . . . . . 12

Figure 4.2 Standoff vs. Uniformity . . . . . . . . . . . . . . . . . . 17

Figure 4.3 Change of Certainty on a Line . . . . . . . . . . . . . . . . 18

Figure 4.4 Least Certain Node Decreases in Certainty . . . . . . . . . . . . 25

Figure 4.5 Relation of Time to Certainty . . . . . . . . . . . . . . 26

Figure 4.6 Time and Certainty . . . . . . . . . . . . . . 27

Figure 4.7 Balance on a Network . . . . . . . . . . . . . . . . . . . . . . 29

Figure 5.1 Two Period Stability . . . . . . . . . . . . . . . . . . . . . . . 32

Figure 5.2 Mean Timesteps to Stability on Pure Opinion Model . . . . . . 33

Figure 5.3 Remodeled Transistor . . . . . . . . . . . . . . . . . . 36

Figure 5.4 Transistor with Support Nodes . . . . . . . . . . . . . . . 40

Figure 5.5 Two Paths, $k$ Transistors . . . . . . . . . . . . . . . . 42

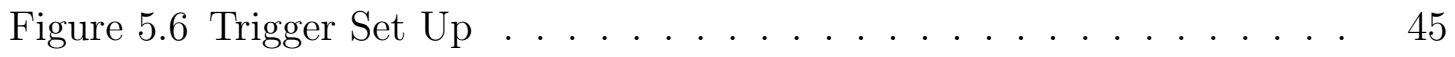




\section{Chapter 1}

\section{Introduction}

The term social network for many is associated with online networks such as Facebook and Twitter, but social networks have been around for as long as humanity, and are defined, by Merriam-Webster, as "network[s] of individuals (such as friends, acquaintances, and coworkers) connected by interpersonal relationships." These relationships make up much of who we are as people. We learn about the wider world first through our relationships, from our parents, then teachers and friends, and later from the bearers of news or gossip.

Psychologists have long studied how peers and social groups influence how people think. In 1956, Solomon Asch did a famous set of experiments demonstrating how subjects, surrounded by a group of people uniformly asserting beliefs that flatly contradict the evidence of the subjects' senses, were induced to conform not only in statement but in belief to the perceived majority opinion [2]. Although followup studies have shown that the percentage of people thus convinced varies with the culture and age of the participants [41, 7], these studies still show the power of the group's perception over the opinions and beliefs of an individual.

Through the study of opinion dynamics, more has been learned not only about how the individual, but also how societies are changed by the structure and composition of social networks. The emergence of online social networks in recent years, along with increased computing capacity in the last half century, has made the study of real-world networks, especially large social networks, easier and more feasible [12]. The spread of ideas and influence has been studied for a range of reasons including its effect on the diffusion of medical and technological innovations, marketing, and political opinion [31, 8, 9, 34, 1, 6, 32, 37].

Two models of social interaction in a network are presented in this paper. Both 
specify mechanics used by individual agents on a graph $G=(V, E)$ to form and spread their opinions. Individuals move to the mass center of all their neighbors in every timestep. In the first model this means an agent's opinion in each timestep is the average weighted opinion of all of its neighbors' opinions in the previous timestep, where the weight of an opinion is determined by the certainty with which the opinion is held. The second model does the same without weights, simply assigning each agent the majority opinion of all of its neighbors. The second model is closely related to the synchronous model described by Frischknecht, Keller, and Wattenhofer in their 2013 paper [22], which will be referred to as the Majority Rules Model. There are slight differences between the second model presented in this paper and the Majority Rules Model in the setup of the networks and the initial steps of the run. Both models were run on 18 networks: real world social networks, of varying sizes, formed on Facebook, as well as artificial networks. The time taken for the models to reach stability on these networks was compared to the bounds proven in this paper and the Majority Rules Model bounds [22]. 


\section{Chapter 2}

\section{Background}

\subsection{Opinion Dynamics}

Opinion Dynamics is a research field which utilizes mathematical and physical models and computational tools, especially the agent-based computational modeling tools, to investigate the diffusion and evolution of opinions in a population of human beings [42]

Opinion dynamics is a wide field spanning many disciplines $[20,23,40,42,28$, $3,37,21,24,46,29,19,17,45,47,31,36,43,30,18]$ with two distinct areas of study. The first subfield examines how agents change their opinion based on the network they are in; the second investigates how social networks change depending on the varying views of the members within them. There is also a range of studies that cross between these two questions to explore how opinion and relationships shift together [45, 39, 46, 4, 24, 38]. This paper will focus primarily on the first subfield, how opinions of agents change and solidify based on the network.

\subsubsection{Influence}

There are a large variety of ways in which agents in a network can form, change, and spread their opinions.

The first paper in the field of opinion dynamics was published by John French in $1956[35,42,20]$ and described a system in which agents slowly change their views to align more closely with the opinions of authority figures. The closer the authority figure's opinion to the agent's opinion, the more sway that authority has over the 
agent. French described two types of influence in this model: direct and indirect. Direct influence exists between two people who are in direct contact; for example, two friends might influence each other's music preferences. Indirect influence occurs when influence travels through several relationships; a friend's older sibling's spouse's cousin might hear a song on the radio and pass it along the chain to the friends. In French's paper, influence only flows in one direction, from the authority figure down, and the convergence of all opinions in the chain to one opinion is inevitable as long as there is one ultimate authority[20].

Since French, most researchers have dealt with networks of bidirectional influence [33, 38]. A model, later called the Flocking Model [11], was published in 2002 by Hegselmann and Krause [26, 27], which expanded on French's model. Although influence in the Flocking Model is bidirectional, no underlying social network exists, meaning that agents do not have a predefined set of neighbors whom they communicate with and are influenced by. Rather, they are influenced by others depending on how close the other agent is in opinion space. Opinion space differs from French's opinion line in that where French allowed agents to agree to varying degrees on only one issue, with the varying opinions represented on an opinion line, opinion space allows for two or more issues to be represented [5].

In 1974, Morris DeGroot built on French's model by adding a trust matrix, a directed and weighted social network. One agent can influence another in proportion to the weight of the edge connecting the first agent to the second, a weight that might be different in the opposite direction. Unlike French's Model and the Flocking Model the degree of influence one node has over another for DeGroot is independent of the similarity of the two agents' opinions. DeGroot's model also differs from the previously discussed models in that it exists on a social network instead of an opinion space [14].

Most models take into consideration the underlying social network, but not every model allows agents to communicate with each of their neighbors in every timestep. In 1997, Robert Axelrod published a paper in The Journal for Conflict Resolution [3] that strove to explain how communication could simultaneously create convergence of opinion and distinct opinion groups. In this model, every agent randomly picks one of its neighbors at every timestep to communicate with [3]. The Deffuant-Weisbuch model extended this limiting of communication, randomly selecting one agent to communicate with one of its neighbors at every timestep [13].

Finally, in 2004 the Galam model introduced a system inspired by a first past the 
post election system. At each timestep subgroups of the overall network "discuss" a binary option. Whichever opinion is more strongly represented in the subgroup is adopted by every member of the group in the next timestep, at which point new subgroups are formed. Serge Galam was specifically looking for conditions under which a minority opinion could spread through a network [23].

\subsubsection{Conformity, Compromise, and Stubbornness}

The second major variable to consider when comparing models in opinion dynamics is the degree to which agents are influenced by their neighbors. In the French, DeGroot, and Flocking models, agents move to the mass center, weighted or unweighted, of every opinion that they encounter $[20,14,5]$, i.e. each agent's opinion becomes the mean opinion of every agent influencing it. This compromising heuristic is a popular choice, but not the only one.

The Deffuant-Weisbuch model also uses the compromise heuristic but only after a certain threshold amount of agreement has been established. If the opinions of two agents differ more than a predetermined threshold value, nothing happens. If the opinions are similar enough, the difference between them is multiplied by some factor and added to the current opinion of both agents[13].

Conformity is the second way in which agents change their opinions. In models employing conformity, an agent overwrites its previous opinion with the opinion of the neighbor influencing it. Axelrod's cultural adoption model uses something between compromise and conformity. Each agent is given a range of opinions on 5 to 15 issues. These opinions determine the agent's culture. While communicating with a neighbor, an agent will adopt one of the previously differing opinions from its neighbor with a probability equal to the cultural similarity of the two agents, conforming completely with the other agent's opinion, but only on one issue [3]. Most models in which agents conform completely are those that seek to track the spread of diseases, a topic that is generally not categorized as opinion dynamics but epidemiology [46].

Finally, a stubborn agent is one which, as introduced by Yildiz in 2010, will not shift its opinions at all[44]. Of course, a network made up of only stubborn agents would not be very interesting, which is why Yildiz and subsequent researchers have examined the effects of varying proportions of stubborn, compromising, and conforming agents existing on the same network [25, 10].

In 2014 an online experiment was conducted in which each participant was asked 
a simple question. Next, the participants were shown fake results, supposedly what other participants had answered to the same question. Finally, the participants were asked the same question again [11]. This experiment clearly is not able to model how individual relationships or reoccurring interactions to opposing opinions tie into the formation of an individual's opinions, however three types of individuals, stubborn, compromising, and conforming, were clearly identifiable. This result lends credibility to the aforementioned studies that modeled these personality types. Interestingly, the number of stubborn participants often depends on the size of the fake group of neighbors shown to be in disagreement [11].

\section{$2.2 \quad$ Information Cascading}

An information cascade is a kind of chain reaction within a social network. The adoption of an opinion or practice by one agent triggers the adoption of the same opinion by other neighboring agents $[15,16]$. There are three main cascading models: the threshold model, the independent cascade model, and the trigger model [31]. In the threshold model, agents randomly select a threshold between 0 and 1 . If the proportion of their neighbors that have adopted some new opinion is above this threshold, the agent also adopts the opinion. In the independent cascade model, agents attempt to convince each of their neighbors of a new opinion with a certain probability of success, but are only able to do so in the timestep after they themselves are convinced [31]. ${ }^{1}$ Finally, in the trigger model, each agent watches a certain set of agents, when one of those agents adopts a new opinion, the watching agent adopts that opinion in the next timestep [31].

\footnotetext{
${ }^{1}$ There are some models, outside of cascading opinions, that explore the reverse of a cascade, where by agents reach out to their neighbors when they feel they do not have enough information instead of being contacted when a new idea comes up $[19,11]$.
} 


\section{Chapter 3}

\section{The Model}

\subsection{The Models}

The original aim in developing the following two models was to gage the effect of confidence weighted opinion on the stabilization of beliefs in a network. Agents in both models move to the mass center of the neighborhood they are in. In the Certainty Model each agent takes each of its neighbors' opinions into account in proportion to that neighbor's confidence. In the Pure Opinion Model each neighbor's opinion is taken into consideration with no weighting of confidence.

Both models work on a graph or network, $G=(V, E)$, of $n$ agents, $V=\{1, \ldots, n\}$ and a set of relationships between agents $E$, which are the edges in the graph $G$. Each agent $i \in V$ has a certainty, $x_{i}(t)$, modeled by a natural number between 0 and 100 , of an opinion $y(t) \in\{0,1, \perp\}$. Before an agent has heard of the issue it has no opinion, $\perp$, and no certainty in that opinion; this represents the initial state of all of the agents.

In the first timestep the population is seeded with $e$ experts, with opinions of 0 or 1 and certainties in those opinions greater than 0 . In this paper only two experts were used. These experts spread their opinions to each of their neighbors who spread the opinions to all of their neighbors, in an independent cascade.

The set $B(t)$ is the set of all nodes on the graph holding the opinion that the more certain expert held in the initial timestep, $t=0$. Conversely, the set $S(t)$ is the set of nodes holding the opinion that the less certain expert held when initially seeding the graph. Without loss of generality, it will be assumed from this point forward that $B(t)$ is the set of nodes holding the 0 opinion and $S(t)$ is the set of nodes holding the 
1 opinion.

To form or change opinion and certainty, agent $i$ will consider the size and certainty of the groups of its neighbors belonging to $B(t)$ compared with those belonging to $S(t)$. Let $N_{i}$ be the open neighborhood of $i$, i.e. the set of neighbors that does not include $i$ and let $\hat{N}_{i}$ be the closed neighborhood, including $i$.

$$
\begin{aligned}
& B_{i}(t)= \begin{cases}\left\{j \in V \mid j \in N_{i} \wedge y_{j}(t)=0\right\} & : y_{i}(t-1)=\perp \\
\left\{j \in V \mid j \in \hat{N}_{i} \wedge y_{j}(t)=0\right\} & : y_{i}(t-1) \neq \perp\end{cases} \\
& S_{i}(t)= \begin{cases}\left\{j \in V \mid j \in N_{i} \wedge y_{j}(t)=1\right\} & : y_{i}(t-1)=\perp \\
\left\{j \in V \mid j \in \hat{N}_{i} \wedge y_{j}(t)=1\right\} & : y_{i}(t-1) \neq \perp\end{cases}
\end{aligned}
$$

If the agent's opinion is newly formed, meaning in the previous timestep the agent had no opinion, then it will not be considered a part of its own neighborhood and will not consider its own opinion, or certainty, in the calculation of its opinion and certainty in the next timestep. After this initial timestep the agent will always be included in the neighborhood, even if its opinion changes.

In the Certainty Model, agent $i$ gains and changes opinions and certainty, based on the opinions and certainties of its neighbors.

$$
y_{i}(t+1)= \begin{cases}0 & : \sum_{j \in B_{i}(t)} x_{j}(t)>\sum_{j \in S_{i}(t)} x_{j}(t) \\ y_{i}(t) & : \sum_{j \in B_{i}(t)} x_{j}(t)=\sum_{j \in S_{i}(t)} x_{j}(t) \\ 1 & : \sum_{j \in B_{i}(t)} x_{j}(t)<\sum_{j \in S_{i}(t)} x_{j}(t)\end{cases}
$$


$x_{i}(t+1)= \begin{cases}\left\lfloor\frac{1}{\left|B_{i}(t)\right|+\left|S_{i}(t)\right|}\left(\sum_{j \in B_{i}(t)} x_{j}(t)-\sum_{j \in S_{i}(t)} x_{j}(t)\right)\right\rfloor & : \sum_{j \in B_{i}(t)} x_{j}(t)>\sum_{j \in S_{i}(t)} x_{j}(t) \\ \left.\mid \frac{1}{\left|B_{i}(t)\right|+\left|S_{i}(t)\right|}\left(\sum_{j \in S_{i}(t)} x_{j}(t)-\sum_{j \in B_{i}(t)} x_{j}(t)\right)\right\rfloor & : \sum_{j \in B_{i}(t)} x_{j}(t)<\sum_{j \in S_{i}(t)} x_{j}(t) \\ x_{i}(t) & : \sum_{j \in B_{i}(t)} x_{j}(t)=\sum_{j \in S_{i}(t)} x_{j}(t)\end{cases}$

In the Pure Opinion Model, agent $i$ gains and changes opinions and certainty in its opinions, based on the number of neighbors which hold each opinion.

$$
\begin{gathered}
y_{i}(t+1)= \begin{cases}0 & :\left|B_{i}(t)\right|>\left|S_{i}(t)\right| \\
y_{i}(t) & :\left|B_{i}(t)\right|=\left|S_{i}(t)\right| \\
1 & :\left|B_{i}(t)\right|<\left|S_{i}(t)\right|\end{cases} \\
x_{i}(t+1)= \begin{cases}\frac{1}{\left|S_{i}(t)\right|+\left|B_{i}(t)\right|}\left|B_{i}(t)\right|-\left|S_{i}(t)\right| & :\left|B_{i}(t)\right|>\left|S_{i}(t)\right| \\
\left|\frac{1}{\left|S_{i}(t)\right|+\left|B_{i}(t)\right|}\right| S_{i}(t)|-| B_{i}(t) \mid & :\left|B_{i}(t)\right|<\left|S_{i}(t)\right| \\
x_{i}(t) & :\left|B_{i}(t)\right|=\left|S_{i}(t)\right|\end{cases}
\end{gathered}
$$

The above equations for calculating opinion are the same as those used in the Majority Rules Model presented by Frischknecht, Keller, and Wattenhofer in 2013. [22] There are three differences between Pure Opinion Model and the Majority Rules model. First, the Majority Rules model does not have any value for certainty. Since certainty does not affect opinion in this model, this change does not affect the result. Second, instead of seeding the network with two experts, the Majority Rules model randomly assigns opinions to each node and, third, since every node starts with an opinion, there is no designated timestep after a node initially gains an opinion in which it does not consider itself part of its own neighborhood. 


\subsection{The Networks}

The models were run on a wide variety of networks which can be split into three different groups.

The first group is composed of a series of structured graphs including a line, a binary tree, a ternary tree, a general tree, and a grid. Each of these was created with 200 nodes.

The second group was comprised of networks artificially generated to mimic social networks. Primarily this means preferential networks, generated by adding nodes to a graph seeded by two connected vertices. For each new node the probability that an edge would be added between it and an existing vertex was relative to the degree of that preexisting vertex over the total possible degree. These networks are of sizes 50, 100, 150, 200, and 250. A random network was also included in this group. The random network was generated by the same process as the preferential networks, but without regard to the degree of each node, instead each node had a one in three chance of forming an edge with any other node. The random network was created with 200 nodes. Each of these networks was created as a connected graph. If, after 200 nodes had been added to the network, not every node was connected, all but the vertices in the largest connected subgraph were removed and the process of adding nodes was repeated until a connected graph was formed.

The third and final group is composed of Facebook groups. Each node represents a person who belonged to that group on Facebook and, as in group three, each edge between the nodes represents a facebook friendship between those two nodes. The sizes of these groups are: 140, 134, 63, 73, 40, 285, and 876. For the purpose of this research in the third group, if unconnected subgraphs existed, all but the largest were removed. No more vertices or edges were added. 


\section{Chapter 4}

\section{Analysis of Certainty Model}

Most of the work done in this field aims to bound the amount of time taken until some sort of consensus is reached on a graph, and to determine the extent and stability of that consensus. [3, 13, 26, 31, 23, 22] In this paper the goal is to stabilize the network and then observe the extent of agreement between the vertices. Stability will be reached when three variables, opinion, certainty, and size of each set $B(t)$ and $S(t)$, have stabilized, meaning they will not change in any future timestep.

Definition 4.0.1. Stability

Stability is a state of the graph at which, between one timestep and the next, nothing changes. Specifically, if the following three conditions are met simultaneously stability has been achieved.

- Stability of number: $\mid(B(t)+S(t)|=|(B(t+1)+S(t+1) \mid$

- Stability of opinion: $\forall v \in G y_{v}(t)=y_{v}(t+1)$

- Stability of certainty: $\forall v \in G x_{v}(t)=x_{v}(t+1)$

\subsection{Tests of Model}

Both of the models described in the previous chapter were run 100 times on each of the 18 graphs previously described, for a total of 1800 tests. In each of these runs, the initially more certain expert had certainty 100 , but the less certain expert initially had certainty 90,60, 40, and 10 in each of 25 runs. Twenty-five pairs of starting points were used for each pair of initial opinions. The starting positions when the lesser 
certainty was 90 were the same as the starting positions when the lesser certainty was 60, 40, and 10 .

Each test was allowed to run until stability was established, which took, on average, 70.1 timesteps; the largest number of timesteps to pass was 393. Below you can see a plot of the average number of timesteps taken by each graph to reach stability.

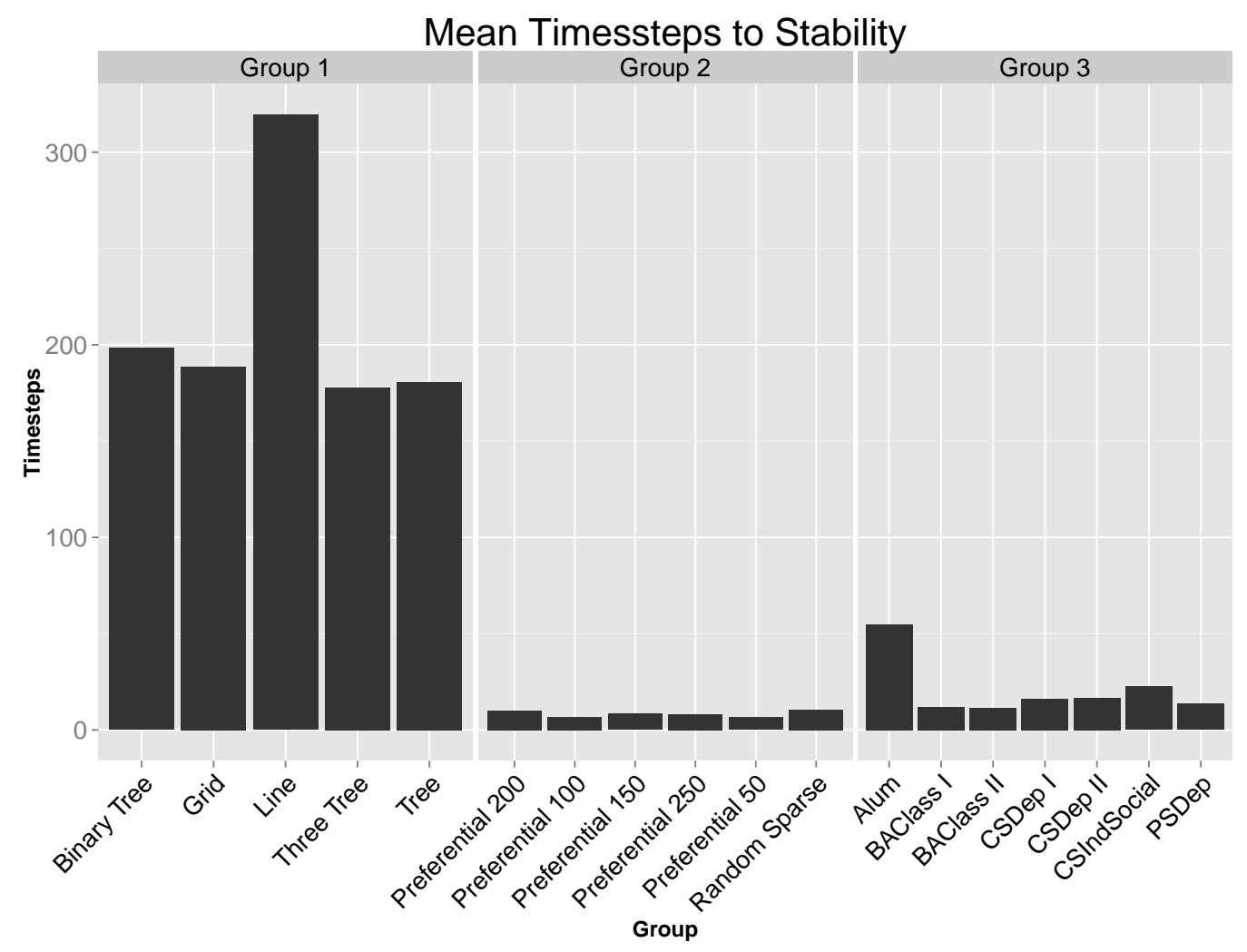

Figure 4.1: Graphs are listed on the x-axis grouped by their type. The y-axis represents the average number of timesteps until stability for each network.

Once stability was reached, the number of timesteps it took to reach stability was recorded. If total consensus, which will be called uniformity, had not been reached, the percentage of nodes holding each opinion was also recorded.

Uniformity when referred to here will indicate that one opinion is held by every vertex, while the other opinion is held by no vertices. The timestep in which uniformity was achieved was recorded for each network that achieved this state, as well as the highest and lowest certainties present in the network at that time. Uniformity occurred in 1328 of the 1800 tests. On lines, trees, and grids, uniformity was reached much less often than on the preferential, random, and social networks. After unifor- 
mity was achieved the average time until stability was 19.5 timesteps (the max was 192).

\subsection{Stability}

Before exploring the topic of length of time until stability, it should be established that a stable state is always attainable. Stability has previously been defined as lack of change between one timestep and the next, with change possible in one of three variables: number, opinion, and certainty.

Lemma 4.2.1. The number of nodes with an opinion will stabilize. That is, at some timestep, $t,|B(t) \cup S(t)|=|B(t+1) \cup S(t+1)|$.

Proof. Proof by contradiction.

Let $N_{B \cup S}$ be the set of all nodes with a neighbor in either the $B$ or $S$.

No node can lose an opinion entirely, meaning the number of nodes with an opinion cannot decrease: $|B(t) \cup S(t)| \leq|B(t+1) \cup S(t+1)|$. Therefore, if the set is to remain unstable in size, the number of nodes with opinions must be continuously increasing. $|B(t) \cup S(t)|<|B(t+1) \cup S(t+1)|$.

The network has a finite number of nodes, $|G| \neq \infty$, meaning there is a limit to the number of nodes that can be added to $B \cup S$ and there will come a point when no more nodes can be added to $B \cup S$. At this point $|B(t) \cup S(t)|$ will not be able to increase or decrease from one timestep to the next and so $|B(t) \cup S(t)|=|B(t+1) \cup S(t+1)|$.

Next, stability of opinion and stability of certainty are strongly linked; a vertex's opinion will only change if the certainty of its neighbors shifts. Therefore, if there is stability of certainty then there must also be stability of opinion.

Lemma 4.2.2. There can be no change in opinion once stability of certainty has been reached.

Proof. If all nodes on the graph have the same opinion, then stability of opinion has been achieved as no node can have a majority of neighbors holding the other opinion. For the rest of the proof therefore it can be assumed that there exist two different opinions on the network. 
Is stability of certainty has been achieved then all nodes will have the equal certainty. Suppose not, and let $v$ be a node for which, $x_{v}(t)>0$ and $x_{v}(t)$ is the maximal certainty on the graph. Let $w(t)$ be a neighbor of $v(t)$ such that $x_{w}(t)<x_{v}(t)$.

The value of $x_{v}(t+1)$ is the mean certainty of all $v$ 's neighbors including itself at time $t$, therefore to ensure $x_{v}(t+1)$ is as large as possible let us assume that, with the exception of $w$, all neighbors of $v$ are as large as possible, that is they are equal to $x_{v}(t)$ which has the maximal certainty in the graph at time $t$.

If $v$ has no neighbors of the opposing opinion:

$$
\begin{aligned}
x_{v}(t+1) & =\left\lfloor\frac{\sum_{u \in \hat{N}_{v}(t)} x_{u}(t)}{\left|\hat{N}_{v}(t)\right|}\right\rfloor \\
& =\left\lfloor\frac{\sum_{u \in \hat{N}_{v}(t) \backslash w} x_{v}(t)+x_{w}(t)}{\left|\hat{N}_{v}(t)\right|}\right\rfloor \\
& <\left\lfloor\frac{\left|\hat{N}_{v}(t)\right| x_{v}(t) \mid}{\left|\hat{N}_{v}(t)\right|}\right\rfloor \text { since } x_{w}<x_{v} \\
& =x_{v}(t)
\end{aligned}
$$

If $y_{v} \neq y_{w}$ :

$$
\begin{aligned}
x_{v}(t+1) & =\left\lfloor\frac{\sum_{u \in \hat{N}_{v}(t) \backslash w} x_{v}(t)-x_{w}(t)}{\left|\hat{N}_{v}(t)\right|}\right\rfloor \\
& <x_{v}(t), \text { since } y_{w} \neq y_{v}
\end{aligned}
$$

This means that stability of certainty implies that all certainties are the same.

Without loss of generality let $y_{v}=0$, remember that nodes with opinion 0 are in set $B$ and nodes of opinion 1 are in set $S$. If all certainties are equal, stable, and greater than 0 then: 


$$
\begin{aligned}
x_{v} & =\left\lfloor\frac{1}{\left|\hat{N}_{v}\right|}\left(\sum_{u \in \hat{B}_{v}(t)} x_{v}-\sum_{w \in S_{v}(t)} x_{v}\right)\right\rfloor \\
x_{v} & =\left\lfloor\frac{1}{\left|\hat{N}_{v}\right|}\left(x_{v}\left(B_{v}|-| S_{v} \mid\right)\right)\right\rfloor \\
1 & =\left\lfloor\frac{1}{\left|\hat{N}_{v}\right|}\left|B_{v}\right|-\left|S_{v}\right|\right\rfloor \\
1 & =\left\lfloor\frac{\left|B_{v}\right|-\left|S_{v}\right|}{\left|B_{v}\right|+\left|S_{v}\right|}\right\rfloor \\
\left|B_{v}\right|+\left|S_{v}\right| & =\left|B_{v}\right|-\left|S_{v}\right| \\
\left|S_{v}\right| & =-\left|S_{v}\right|
\end{aligned}
$$

Therefore if there are more than two opinions on a graph and stability of certainty has been achieved the certainty of all nodes must be 0 .

If the certainty of all nodes is 0 no opinion can change as no set of neighbors can have a higher cumulative certainty than another.

Lemma 4.2.3. The certainty of all nodes on the graph will stabilize.

The following two Lemmas are needed to prove 4.2 .3 but will be proved themselves in sections 4.4 and 4.6 .

Lemma 4.2.4. Once there no longer exist two opinions on the graph a stability of certainty will be reached within $O(d)$ timesteps.

Lemma 4.2.5. Assuming stability of number has been established and not all nodes have the same opinion, it will take no more than $O(d)$ timesteps to reach stability.

Proof of Lemma 4.2.3. Lemma 4.2.4 shows that if all nodes share a common opinion stability of certainty will be reached in $O(d)$ timesteps. Lemma 4.2 .5 shows that the same limit applies if there exist two opinions on the graph. 
Since there can never be more than two opinions on a graph nor can there be fewer than one opinion on a graph stability of certainty will be reached in every case.

\subsection{Uniformity and Standoff}

Definition 4.3.1. A graph is in a uniform state if it is stable with respect to opinion, and every node holds the same opinion. That is, either $|S|=0$ or $|B|=0$.

Definition 4.3.2. A graph is in a state of standoff when both opinions are represented, i.e. $|B| \geq 1$ and $|S| \geq 1$ and stability of certainty has been attained.

Since we start with $|B|=|S|=1$ and it is never possible for a node to revert to having no opinion, standoff and uniformity are the only two possibilities once stability has been reached. ${ }^{1}$

Empirical Observations: Of the 1800 tests described in section 4.1, 471 reached a stability in a state of standoff and 1328 gained uniformity. Figure 4.2 is a breakdown by the type of network of what percentage reached uniformity versus standoff. The average number of timesteps to stability on a uniform network was 23.2, while standoff was achieved within an average of 202.2 timesteps.

In many cases the position of the initial nodes will determine whether uniformity or standoff will be reached. However, as seen in Figure 4.2, uniformity is more likely on some graphs than others and without knowledge of the position of the initial nodes it is still possible, through analysis of the general graph structure and the initial certainties of each opinion, to determine the likelihood of uniformity or standoff. The tests show, for example, that tests on the line, trees, grid, rarely, if ever, reached uniformity, though it is possible on each of these graphs.

\footnotetext{
${ }^{1}$ Note that uniformity is a state of a network which may or may not have achieved stability of certainty where as standoff refers to a state of stability in opinion and certainty.
} 


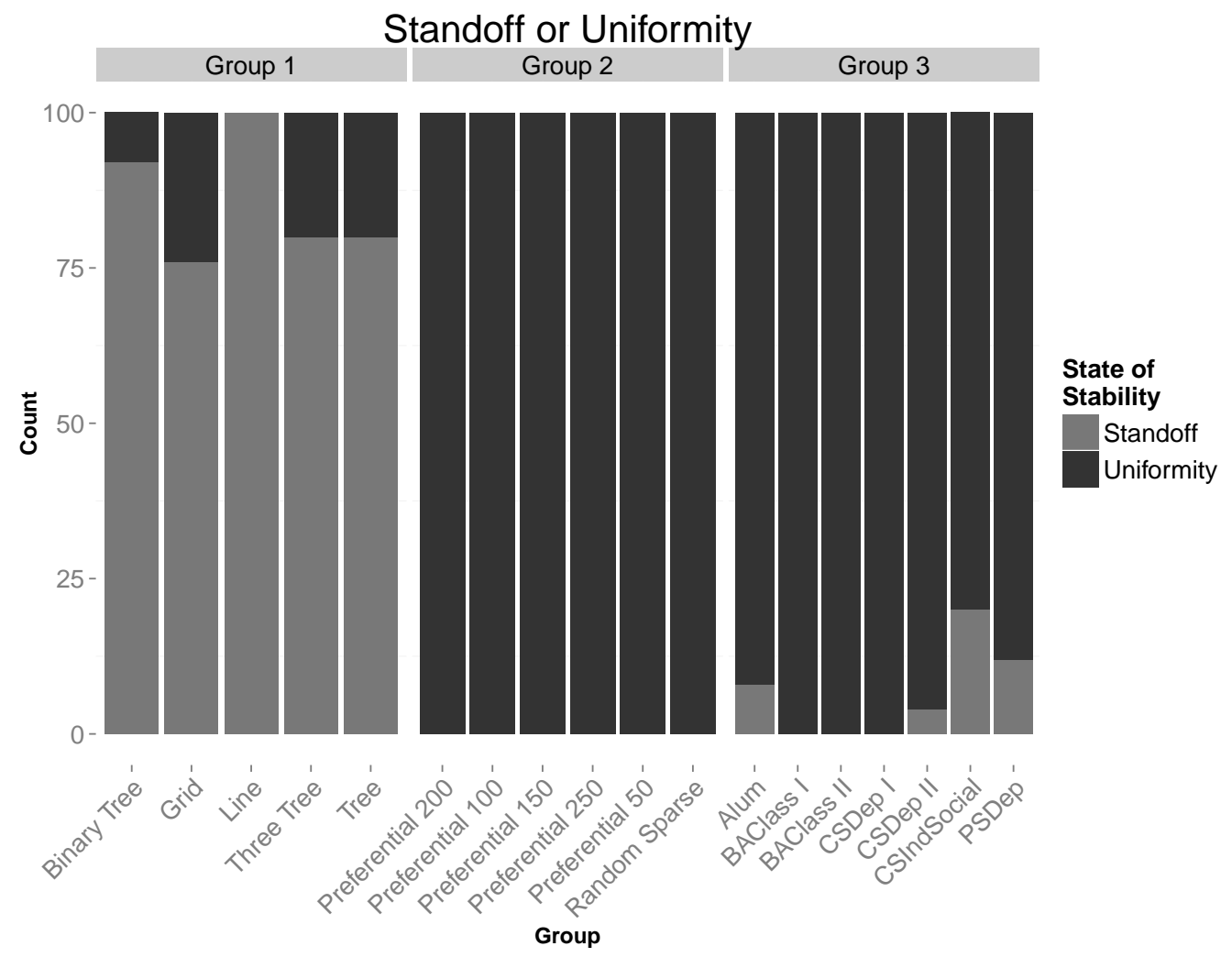

Figure 4.2: The number of trials that resulted in a uniform opinion are represented in black, while those resulting in a standoff are in gray. Trials are broken down by graph and split into three groups: classically constructed graphs, preferential and random networks, and social networks.

Observe that on a line, each time a non-leaf node is converted from one opinion to the other, the certainty of the node being converted, $b$, has at most one third of the certainty of the node, $a$, which it was converted by; $x_{b}(t+1) \leq \frac{x_{a}(t)}{3}$. See figure 4.3 .

This reduction is relevant as it means that certainty decreases quickly as an opinion travels along the line. If the larger certainty doesn't reach the end of the line before it becomes too small to convince nodes of its opinion, uniformity will not be reached.

In trees the same logic applies, however a tree with the same number of nodes as a line will have a smaller diameter and therefore the larger certainty will on average have to travel less far to reach the end, increasing the likelihood that uniformity is reached. However, trees are not significantly more likely to achieve uniformity as the certainty of the node convincing must be higher if the node to be convinved has more than one neighbor that agrees with it. 


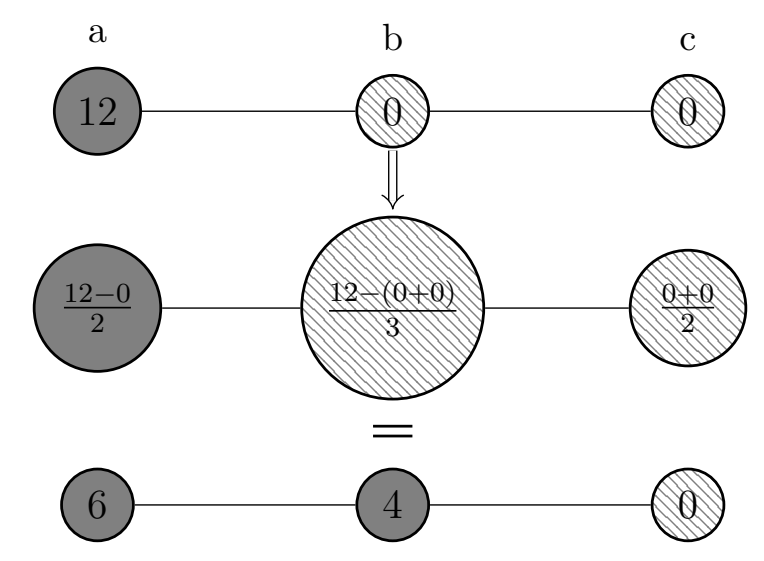

Figure 4.3: Change of certainty on a line.

In more dense graphs, such as grids, the diameters of the graphs are smaller again, meaning there are fewer timesteps in which the convincing certainty is decremented. A convincing node may also have allies, nodes of the same opinion which are also neighbors to the node being convinced. These allies may allow the certainty of the convincing nodes to be lower than in tree or line graphs. This cuts both ways however, and, as in trees, the nodes being convinced may have neighbors supporting their opinion, requiring a higher certainty in the convincing nodes.

Generally then it can be observed that more dense graphs are more likely to achieve uniformity. This will be discussed further in section 4.5 once the time to stability has been considered.

\subsection{Stable Uniformity}

When a graph reaches stabilized uniformity there are two distinct steps: first, the time at which there cease to be two opinions represented in the network and second, stability of certainty. Both of these steps take $O(d)$ time where $d$ is the diameter of the graph. For simplicity the bound for stability of certainty will be established before the bound on time taken until uniformity.

\subsubsection{Time from Uniformity to Stability}

Let $L(t)$ be the set of nodes with maximum certainty in $G$ at time $t$. This certainty will be denoted $x_{L}(t)$. Similarly let $M(t)$ be the set of nodes with the minimum 
certainty in $G$ at time $t$, certainty denoted $x_{M}(t)$.

Lemma 4.4.1. If $v \in L(t)$ and $\exists w \in N_{v}$ such that $w \notin L(t)$, then $x_{v}(t)>x_{v}(t+1)$. Proof. To make $x_{v}(t+1)$ as large as possible let us assume that with the exception of $w$ all other neighbors of $v$ are as large as possible, that is they are all in $L(t)$.

If $v$ has no neighbors with a different opinion:

$$
\begin{aligned}
x_{v}(t+1) & =\left\lfloor\frac{\sum_{u \in \hat{N}_{v}(t)} x_{u}(t)}{\left|\hat{N}_{v}(t)\right|}\right\rfloor \\
& =\left\lfloor\frac{\sum_{u \in \hat{N}_{v}(t) \cap L(t)} x_{L}(t)+x_{w}(t)}{\left|\hat{N}_{v}(t)\right|}\right\rfloor \\
& <\left\lfloor\frac{\left|\hat{N}_{v}(t)\right| x_{L}(t) \mid}{\left|\hat{N}_{v}(t)\right|}\right\rfloor \\
& =x_{L}(t) \\
& =x_{v}(t)
\end{aligned}
$$

If $y_{v} \neq y_{w}$ :

$$
\begin{aligned}
x_{v}(t+1) & =\left\lfloor\frac{\sum_{u \in \hat{N}_{v}(t) \cap L(t)} x_{L}(t)-x_{w}(t)}{\left|\hat{N}_{v}(t)\right|}\right\rfloor \\
& =x_{v}(t), \text { since } y_{w} \neq y_{v}
\end{aligned}
$$

Lemma 4.4.2. If $w \notin L(t)$ but $w \in N_{L(t)}$, then $x_{w}(t+1)<x_{L}(t)$.

Proof. The way in which nodes increase their certainty is by being influenced by more certain neighbors, however even assuming all of $w$ 's neighbors have the highest certainty in the graph, if $w \notin L(t)$, then $w \notin L(t+1)$ if $x_{L}(t)=x_{L}(t+1)$. 


$$
\begin{aligned}
x_{w}(t+1) & =\left\lfloor\frac{\sum_{u \in \hat{N}_{w}(t)} x_{u}(t)}{\left|\hat{N}_{w}(t)\right|}\right\rfloor \\
& =\left\lfloor\frac{\left(\sum_{u \in\left(N_{w}(t)\right) \cap L(t)} x_{L}(t)\right)+x_{w}(t)}{\left|\hat{N}_{w}(t)\right|}\right\rfloor \\
& <\left\lfloor\frac{\left|\hat{N}_{w}(t)\right| x_{L}(t) \mid}{\left|\hat{N}_{w}(t)\right|}\right\rfloor \\
& =x_{L}(t)
\end{aligned}
$$

Lemma 4.4.3. After Uniformity is reached, the largest certainty on a graph will not increase. That is, $x_{L}(t) \leq x_{L}(t+1)$.

Proof. No node, $v$, will have a greater certainty in timestep $t+1$ than $x_{L}(t)$.

$$
\begin{aligned}
x_{v}(t+1) & =\left\lfloor\frac{\left.\sum_{u \in \hat{N}_{v}(t)} x_{u}(t)\right\rfloor}{\left|\hat{N}_{v}(t)\right|}\right\rfloor \\
& =\left\lfloor\frac{\sum_{u \in\left(\hat{N}_{v}(t)\right) \cap L(t)} x_{L}(t)}{\left|\hat{N}_{v}(t)\right|}\right\rfloor, \text { assume } \forall u \in N_{v}, u \in L(t) \\
& =x_{L}(t)
\end{aligned}
$$

Now that it is clear that certainties will not increase past $x_{L}(t)$ and that, while $|L(t)| \neq|G|,|L(t)|>|L(t+1)|$ or $x_{L}(t)>x_{L}(t+1)$, it is left to be shown how fast $|L|$ will decrease and at what point stability or uniformity will be reached. 


\section{Definition 4.4.1.}

$d_{v}^{\prime}(t)= \begin{cases}0 & : \text { if } v \notin L(t) \\ \text { length of the shortest path from } v \text { to any node not in } L(t) & : \text { if } v \in L(t)\end{cases}$

The longest stortest path, $d^{\prime}(t)$ is the $\max d_{v}^{\prime}(t)$ for all $v \in G$.

Lemma 4.4.4. Assuming uniform opinion and $|L(t)| \neq|G|$, every node in $L(t)$ will decrease in certainty within d timesteps, at which point $x_{L}$ will decrease.

Proof. $G$ is a connected graph, therefore if $|L(t)| \neq|G|, d^{\prime}(t)$ is finite; let $v$ be a vertex such that $d_{v}^{\prime}=d^{\prime}(t)$.

Let $P$ be the longest shortest path and let $v$ be a node on $P$.

By 4.4.2, we know that $x_{v}(t+1)<x_{L}(t)$ and therefore $|P(t)|=|P(t+1)|+1$.

Since $|P|$ reduces by 1 in every timestep until $|P|=0$ and $P(t)$ represents the shortest path between its endpoint in $L(t)$ and a node $w \notin L(t)$, it will take at least $|P(t)|-2$ timesteps for this endpoint to have a neighbor not in $L(t)$. Because $P(t)$ also represents the longest path between any node in $L(t)$ and a node not in $L(t)$ it will also take at most $|P(t)|$ timesteps for $x_{L}$ to decrease. Therefore, it will take $|P(t)|$ timesteps for $x_{L}$ to decrease.

Since the length of $P(t)$ can be at most the diameter, $d$, of the graph, $x_{L}$ will decrease in at most $d$ timesteps.

Observe that the more nodes in the set of $L(t)$ with neighbors outside the set, the shorter $P$ is likely to be.

The preceding four Lemmas have primarily dealt with the nodes of the greatest certainty on the graph. The following will set a limit to how far these certainties will decrease by examining the nodes of least certainty.

Lemma 4.4.5. In no time after $t$ will any certainty be less than $x_{M(t)}$

Proof. For $x_{w}(t+1)$ to be as small as possible $\hat{N}_{w} \subseteq M(t)$. 


$$
\begin{aligned}
x_{w(t+1)} & =\left\lfloor\frac{\left.\sum_{u \in \hat{N}_{w}} u\right\rfloor}{\left|\hat{N}_{w}\right|}\right\rfloor \\
& =\left\lfloor\frac{\sum_{u \in\left(\hat{N}_{v}(t)\right) \cap M(t)} x_{M}(t)}{\left|\hat{N}_{w}(t)\right|}\right\rfloor, \text { assume } \forall u \in N_{v}, u \in M(t) \\
& =x_{M}(t)
\end{aligned}
$$

This brings us to the proof of Lemma 4.2.4, which states Once there no longer exist two opinions on a graph stability of certainty will be reached within $O(d)$.

Proof of Lemma 4.2.4. Lemma 4.4.4 showed that all nodes in $L(t)$ set will decrease in certainty within $d$ timesteps. If, after $x_{L}$ has decreased, stability has not been achieved there will be a new $L$ such that $|L(t)|<|G|$. This new $L(t)$ will decrease in, at most, $d$ timesteps and the process will continue to repeat until stability is achieved.

Lemma 4.4.5 showed that there is a lower bound to how low the stable certainty will decrease, $x_{L}$ can only decrease until it reaches this lower bound. If nodes in $L$ hold every certainty between $x_{L}$ and this lower bound, $L$ will decrease in certainty $r$ times where $r$ is the difference between $x_{L}$ and $x_{M}$.

Therefore we know that the most timesteps until stability is $d(r-1)$, which gives us an upper bound of $\mathrm{O}(\mathrm{d})$ when we consider that $r$ can be at most 100 .

\subsubsection{Time until Uniformity is Reached}

It should be acknowledged that while it is more likely that the set of nodes with the initially larger opinion, $B$, will be the set present on the network once uniformity is reached, this is not always the case. In the tests described in section 4.1, of the 1328 runs that resulted in stable uniformity, 1304 uniformly held the $B$ opinion and 24 held the $S$ opinion. For the following analysis however, without loss of generality, it will be assumed that the $S$ opinion will disappear from the network. 
Let $L_{S}$ be the set of nodes in $S$ for which there does not exist a node in $S$ with a larger certainty.

Theorem 4.4.6. Once stability of number is reached, the number of timesteps until uniformity is achieved will be $O(d)$.

Proof. $L_{S}(t)$ will reduce in size or certainty at every subsequent timestep as long as $\left|L_{S}\right| \neq|S|$. The reason for this will be much the same as proved in Lemmas 4.4.1 and 4.4.3. If there exists a node $v \in L_{S}(t)$ with one or more neighbors outside of $L_{S}(t)$, then $L_{S}(t)$ will either decrease in size or certainty in the next timestep. The difference is, not all neighbors of $L_{S}(t)$ must have certainty below the certainty of the nodes in $L_{S}(t)$ and it is possible that a node that leaves $L_{S}(t)$ will not be part of $L_{S}$ again, regardless of how low $x_{L_{S}}$ becomes, as the node may have changed opinion.

The time until $x_{L_{S}}$ reduces will again be at most the diameter of the graph, but the lowest $x_{L_{S}}$ will reduce to, is zero, rather than the minimum certainty on the network. Unlike the certainties of nodes once uniformity is established, which will only reduced if they had a neighbor of lesser certainty, while there remain two opinions on the network a node's certainty can decrease even if it is the least certain node in the network, as long as it has a neighbor of the opposite opinion.

Since it is not clear which opinion will be represented on the uniform network it will be the largest certainty, not the largest certainty of one particular subset, that will be multiplied by the diameter of the network to find to worst case time until uniformity is reached, $x_{G} \mathrm{~d}$, however since $x_{G}$ can be no larger than 100 the limit is $O(d)$. 


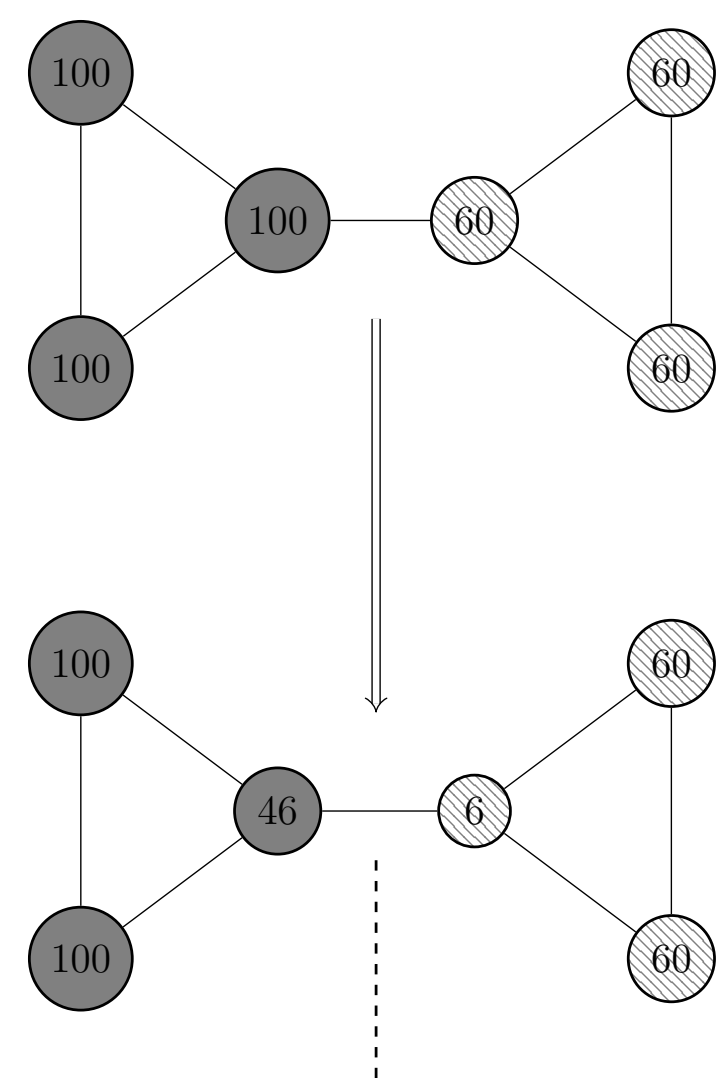

Later in the run.

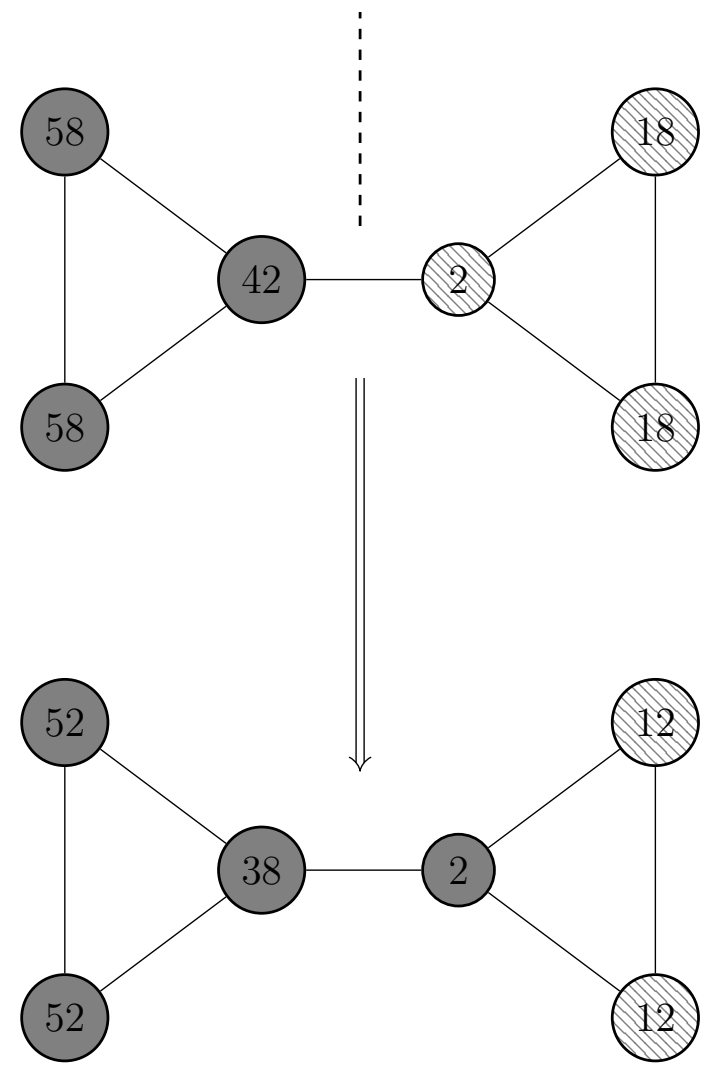

$\left|L_{S}\right|$ decreases because of a neighbor with an opposing opinion.

\footnotetext{
$x_{L_{S}}$ decreases because of a neighbor in $\mathrm{S}$ with lesser value. $|S|$ decrease because of a neighbor of the opposing opinion.
} 


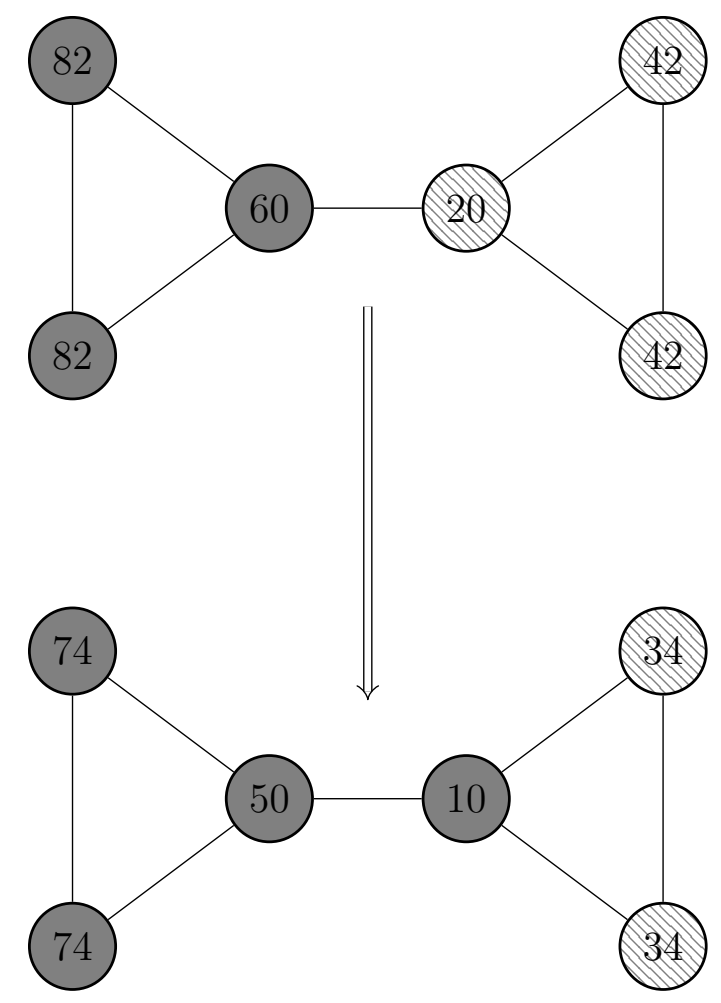

At another point in the run of 100-60 network seen in the previous figure. The certainty of the least certain node decreases.

Figure 4.4: Least Certain Node Decreases in Certainty when Confronted with Opposing Opinion.

\subsection{Certainty of a Uniform Stable Graph}

Figure 4.5 shows the certainty and the timestep at which every test described in section 4.1 stabilized and there is an observable correlation between the two variables. As the timesteps increase there are fewer points representing high certainties, the tests that took the most timesteps do not reach any certainty higher than zero.

This relationship between timestep and maximum possible certainty is also reflected in the proofs of the Lemmas in section 4.4 which used the fact that after at most $d$ timesteps the node or nodes with the largest certainty in the graph would decrease in certainty. The more timesteps before stability the greater number of timesteps in which $x_{L}$ might decrease.

This effect can be seen clearly once uniformity is reached. In Figure 4.6 the number of timesteps from the disappearance of the second opinion to the timestep in which stability is gained is charted against the change in the average certainty between those timesteps. Notice that regardless of the certainties of the original experts the change in certainty remains tied to the number of timesteps. 


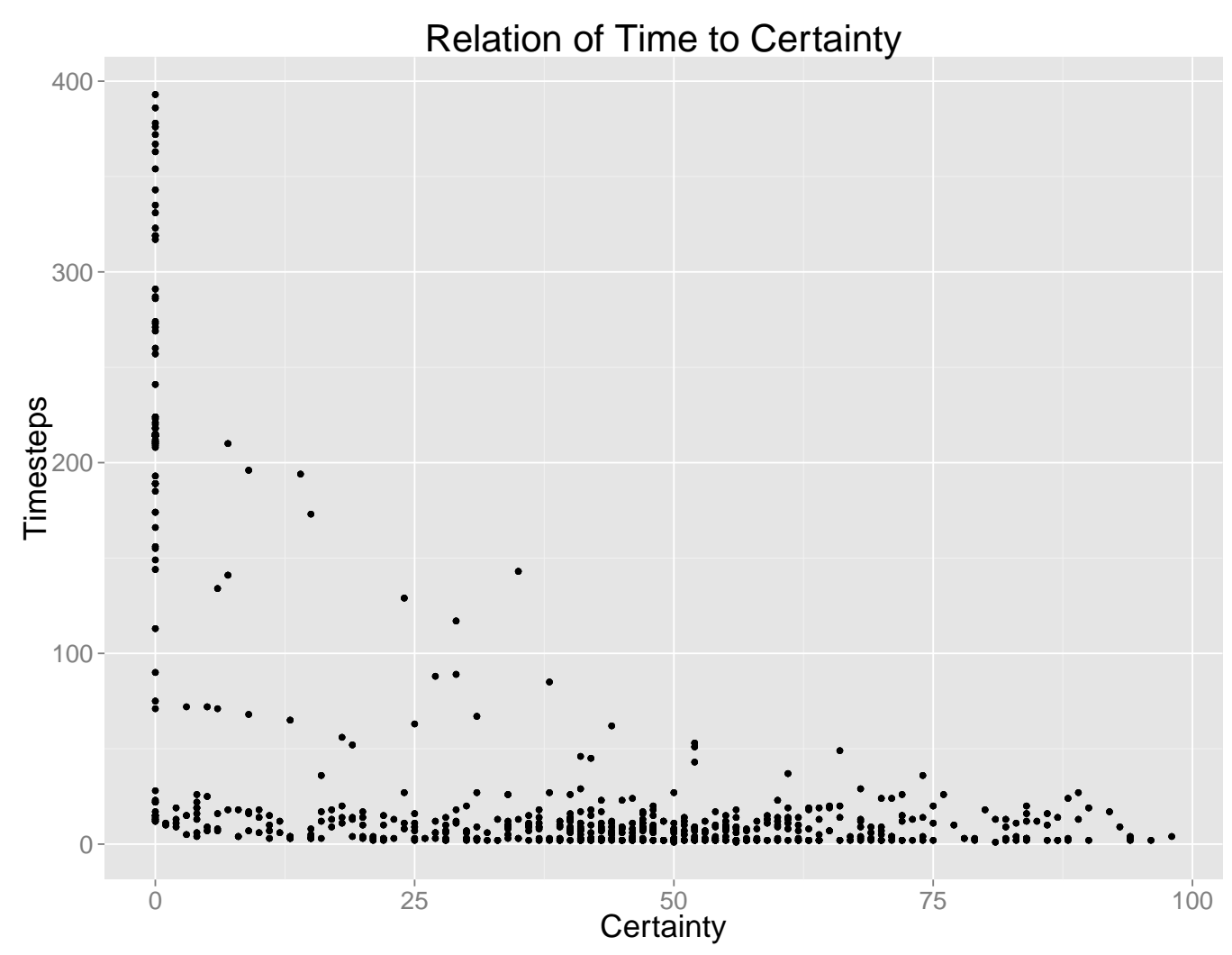

Figure 4.5: Each point represents the certainty of a test in the timestep after uniform stability is achieved.

\subsection{Stable Standoff}

Theorem 4.6.1. A standoff will be reached in $O(d)$ time and at this time the certainty of every node will be zero.

The proof of this theorem will be in two parts, first that the certainty of every node will be 0 once stability is reached and second that it will take no more than $O(d)$ timesteps for stability to be established.

Lemma 4.6.2. All nodes on a stable network with two different opinions represented must have certainty 0 .

Proof. Stability of certainty means $\forall v \in G x_{v}(t)=x_{v(t+1)}$.

By definition of standoff and a connected graph, $\exists v, w \in G$ such that $y_{v} \neq y_{w}$ and $v w \in E$. 


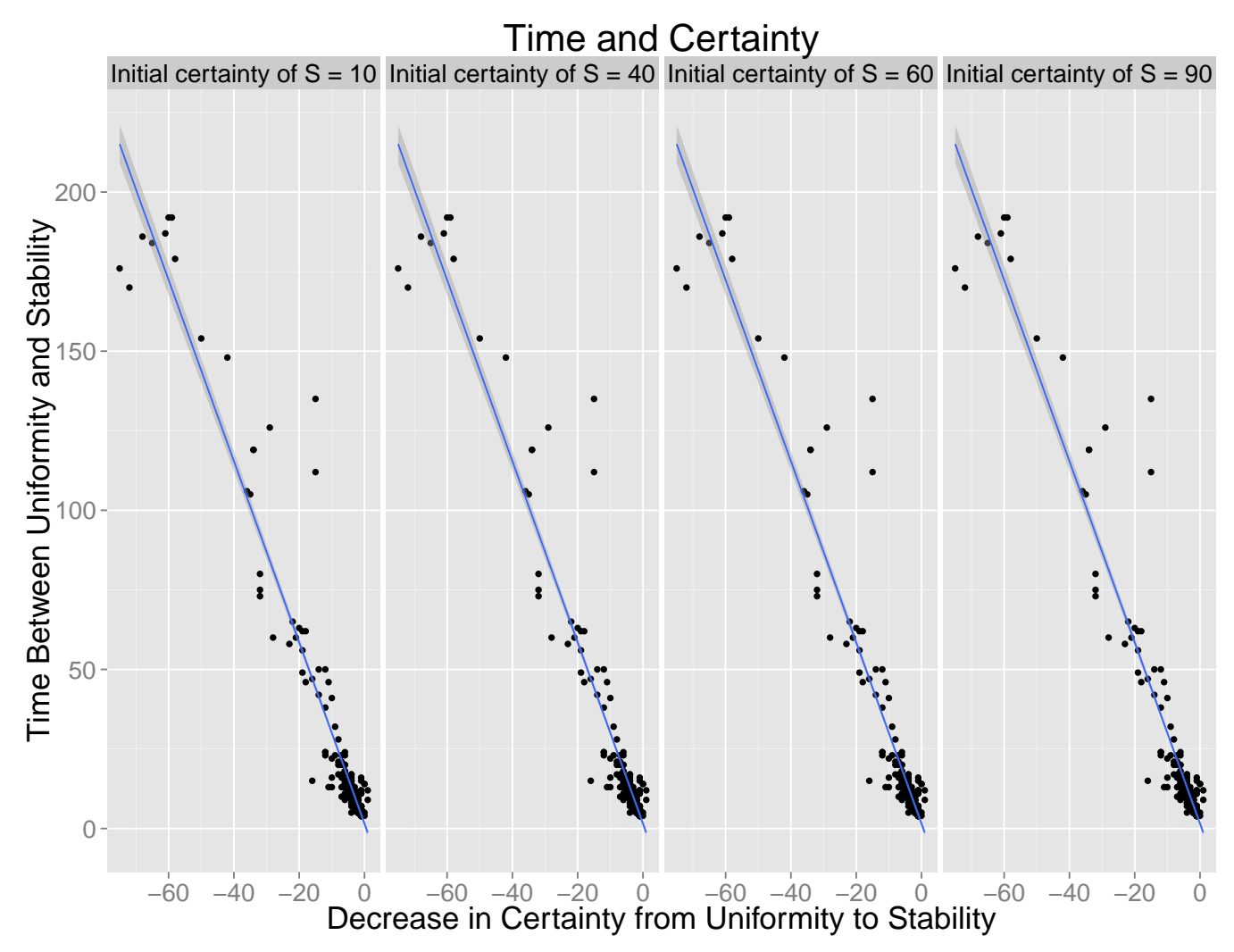

Figure 4.6: Each plot is labeled at the top with the initial certainty of $S$. In each run the certainty of $B$ was 100. Each node is colored to represent the amount of clustering in the graph.

$$
\begin{aligned}
x_{v}(t+1) & =\left\lfloor\frac{1}{\left|\hat{N}_{v}\right|}\left(\sum_{u \in \hat{N}_{v}(t) \mid y_{u}=y_{v}} x_{u}(t)-\sum_{u \in N_{v}(t) \mid y_{u} \neq y_{v}} x_{u}(t)\right)\right\rfloor \\
& =x_{v}(t+1), \text { can only be true while } v w \in E \text { if } x_{u}(t)=0
\end{aligned}
$$

If there exists $L_{G}(t)$ such that $x_{L_{G}}(t)>0$ then there must be a node $w \in N_{L_{G}}(t)$ such that $w \notin L_{G}(t)$.

By Lemma 4.4.1 $x_{v}$ will decrease for every $v \in L_{G}(t) \cap N_{w}$ meaning if exists $L_{G}(t)$ such that $x_{L_{G}}(t)>0$ the network cannot be stable.

Now Lemma 4.2.5, Assuming stability of number has been established and not 
all nodes have the same opinion, it will take no more than $O(d)$ timesteps to reach stability, will be proved.

Proof of Lemma 4.2.5. If $|L(t)| \neq|G|$, then $\exists v, w \in G$ such that $v \in L(t), w \notin L(t)$, and $v w \in E$.

If $y_{v} \neq y_{w}$, then:

$$
\begin{aligned}
x_{v}(t+1) & =\left\lfloor\frac{1}{\left|\hat{N}_{v}\right|}\left(\sum_{u \in \hat{N}_{v}(t) \mid y_{u}=y_{v}} x_{u}(t)-\sum_{u \in N_{v}(t) \mid y_{u} \neq y_{v}} x_{u}(t)\right)\right\rfloor \\
& =\left\lfloor\frac{1}{\left|\hat{N}_{w}\right|}\left(\sum_{u \in \hat{N}_{v}(t) \mid y_{u}=y_{v}} x_{L_{B}}(t)-\sum_{u \in N_{v}(t) \mid y_{u} \neq y_{v}} x_{u}(t)\right)\right\rfloor \\
& <x_{v}(t), \text { since } x_{w} \geq 0
\end{aligned}
$$

If $y_{v}=y_{w}$ then by 4.4.1 $x_{v}(t)>x_{v}(t+1)$.

Therefore as long as there exists a node in $L$ with a neighbor not in $L$, that is while $|L(t)| \neq|G|$, the certainty of some node on the network will decrease in every timestep.

By 4.4.4 it will take $O(d)$ timesteps for $x_{L}$ to decrease, which will happen at most $x_{L}$ times, since $x_{L}$ can be at most 100 , stability of certainty will be reached in $O(d)$ time.

Remember that by Lemma 4.2.2 once stability of certainty is reached the graph will also have stability of opinion. Therefore after stability of number is reached, if not all nodes hold the same opinion, stability will be reached in $\mathrm{O}(\mathrm{d})$ timesteps.

\subsection{Stability of Number}

In most cases stability of number is easily predicted. In each timestep nodes without an opinion who have neighbors with an opinion, become part of either $B$ or $S$ and the graph fully stabilizes within $d$ timesteps. The one case in which this is not true is when a balanced vertex exists on the network.

Definition 4.7.1. A node $v$, is balanced when $\sum_{j \in B_{v}(t)} x_{j}=\sum_{j \in S_{v}(t)} x_{j}$. 
Balance may delay the progression towards stability of number if the node does not yet have an opinion. For example, as shown in figure 4.7, if a node has two neighbors of one opinion with certainty 30 , and another neighbor of the opposing opinion with certainty 60 , in the next timestep, since neither opinion's certainty outweighs the other, the node in question will not be convinced of either opinion. This may be a permanent balance, in which case stability of number will not have been delayed, or it may be a temporary balance, broken when one neighbor changes certainty.
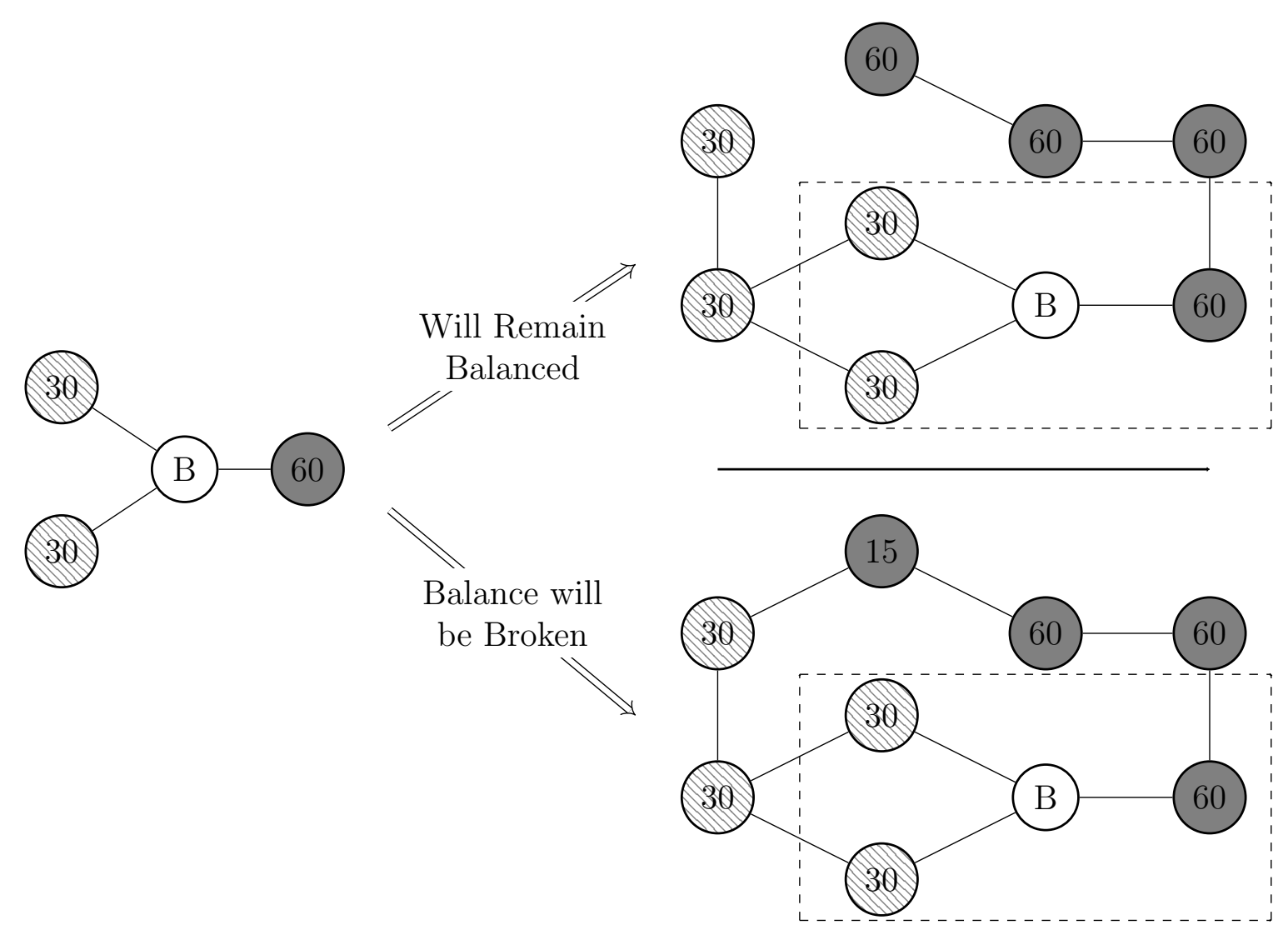

Figure 4.7: The subgraph to the left can be part of either network pictured with different results depending on the existence of a single edge.

Lemma 4.7.1. If all balanced nodes are removed from $G$ and nodes in $S$ and $B$ are no longer connected, then balance will never be broken.

Proof. For the balance of node $v$ to be broken at some $t$ either $\sum_{j \in B_{v}(t-1)} x_{j}>\sum_{j \in S_{v}(t-1)} x_{j}$ or $\sum_{j \in B_{v}(t-1)} x_{j}<\sum_{j \in S_{v}(t-1)} x_{j}$, meaning the certainty of one or more of $v$ 's neighbors must change for balance to be broken. 
Nodes only change certainty when met with an opposing opinion or a lesser certainty. Therefore until nodes of one opinion meet with a node of the opposing opinion all nodes of both opinions will have the same certainty.

$$
\begin{aligned}
x_{w}(t+1) & =\left\lfloor\frac{1}{\left|\hat{N}_{w}\right|}\left(\sum_{j \in \hat{N}_{w}(t) \mid y_{j}=y_{w}} x_{j}(t)-\sum_{j \in N_{w}(t) \mid y_{j} \neq y_{w}} x_{j}(t)\right)\right\rfloor, \text {, since } S \text { and } B \text { are disconnected } \\
& =\left\lfloor\frac{1}{\left|\hat{N}_{w}\right|}\left(\sum_{j \in \hat{N}_{w}(t) \mid y_{j}=y_{w}} x_{j}(t)-0\right)\right\rfloor, \text { since all } x_{j}(t) \text { are the same } \\
& =\left\lfloor\frac{1}{\left|\hat{N}_{w}\right|}\left(\sum_{j \in \hat{N}_{w}(t) \mid y_{j}=y_{w}} x_{w}(t)-0\right)\right. \text { are the same }
\end{aligned}
$$

If nodes never change certainty, balance cannot be broken.

In figure 4.7 balance is broken as soon as the decreased certainty from the converted node travels back to the balanced node, that is four timesteps after the uppermost node gains certainty 15. If, in figure 4.7 the original certainties were 32 and 16 , it is the second timestep after the reduced certainty reaches the point of balance that the node is converted. In theory a configuration might be found in which balance is maintained for a third and fourth timestep, however there is a bound to the number of timesteps this can continue.

Lemma 4.7.2. It will take $O(n)$ timesteps for balance to be broken if it can be broken. Proof. Let $G^{\prime}$ be the subgraph of $G$ that does not include the balanced nodes.

If $G^{\prime}$ stabilizes uniformly then, by the definition, all nodes will hold the same opinion. Once all nodes hold the same opinion in $G^{\prime}$, balance will be broken on the balanced nodes in $G$. This will happen in no fewer than $d^{\prime}$ timesteps by Theorem 4.4.4.

If $G^{\prime}$ reaches stability in a state of standoff, by Theorem 4.6.1 we know that every node will change its certainty until it reaches 0 in $d^{\prime}$ timesteps. If balance in $G$ is not 
broken by the time $G^{\prime}$ is stable, balance will not be broken because after stability is reached in $G^{\prime}$ no node with the ability to break the balance will change.

In either case it will take $O\left(d^{\prime}\right)$ timesteps for stability of number to be reached. As $d^{\prime}$ can be no larger than $n-1$ stability of number will be achieved in $O(n)$ time. 


\section{Chapter 5}

\section{Analysis of Pure Opinion Model}

Stability for the Pure Opinion Model is similar to stability for the Certainty Model with two key differences. First, certainty is no longer a factor in determining the stability of a system. The certainty of vertex $v$ simply reflects the percentage of neighbors that hold the same opinion as $v$ and does not change the opinion or certainty of $v$ or any of $v$ 's neighbors. Second, stability for the Pure Opinion Model can be periodic, between one timestep and the next nodes may change their opinions as long as at some future timestep all the same nodes have the same opinions and then change them again in the same order. Figure 5.1 is an example of a two period stability.
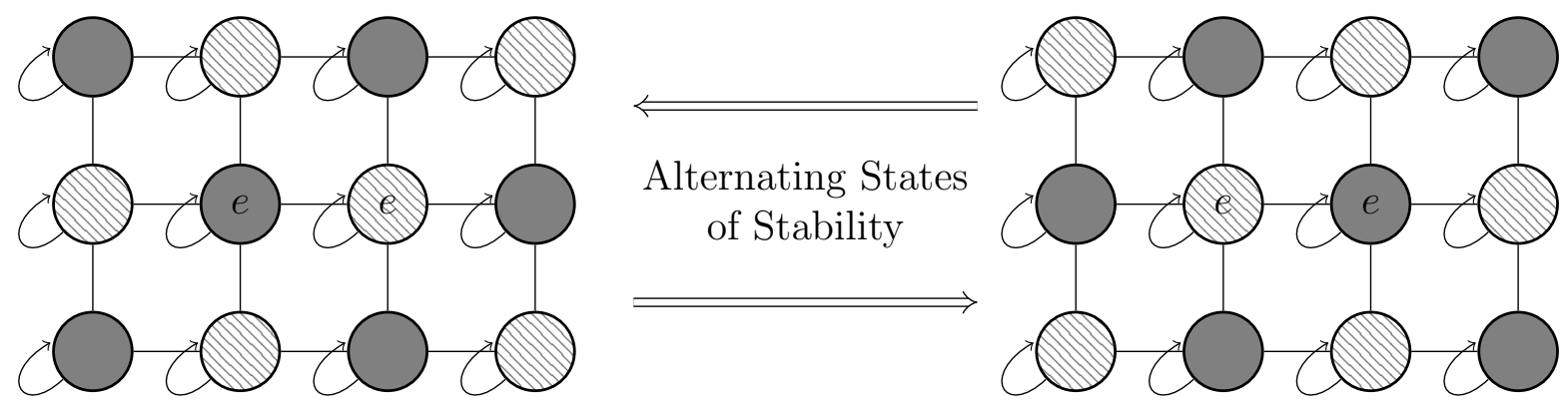

Figure 5.1: Each opinion is represented by a different color and the two nodes labeled $e$ are the initial experts holding these opinions; it doesn't matter which expert held which opinion at time 0 .

In 2013 Frischknecht, Keller, and Wattenhofer, who created the very similar Majority Rules Model, proved an almost tight bound for the maximum number of periods possible in a system, which was 2. [22]

Definition 5.0.2. Stability is a state of the graph at which there is no deviation from 
a set pattern of opinions being held from one timestep to the next. Specifically both of the following are true:

- Stability of number: $\mid(B(t)+S(t)|=|(B(t+1)+S(t+1) \mid$

- Stability of opinion: $\forall v \in G,\left\{y_{v}(t), y_{v}(t+1), \ldots, y_{v}(t+l)\right\}=\left\{y_{v}(t+l), y_{v}(t+\right.$ $\left.l+1), \ldots, y_{v}(t+l+l)\right\}$, where $l$ is the period of stability.

The Pure Opinion Model was run on each of the 18 networks described in Chapter 2. Twenty-five pairs of starting points were chosen at random for each network and each test was run until stability was achieved. The figure 5.2 shows the average time to stability on each of the 18 networks. In each case the stability reached was a one period stability, that is every timestep was the same as the last.

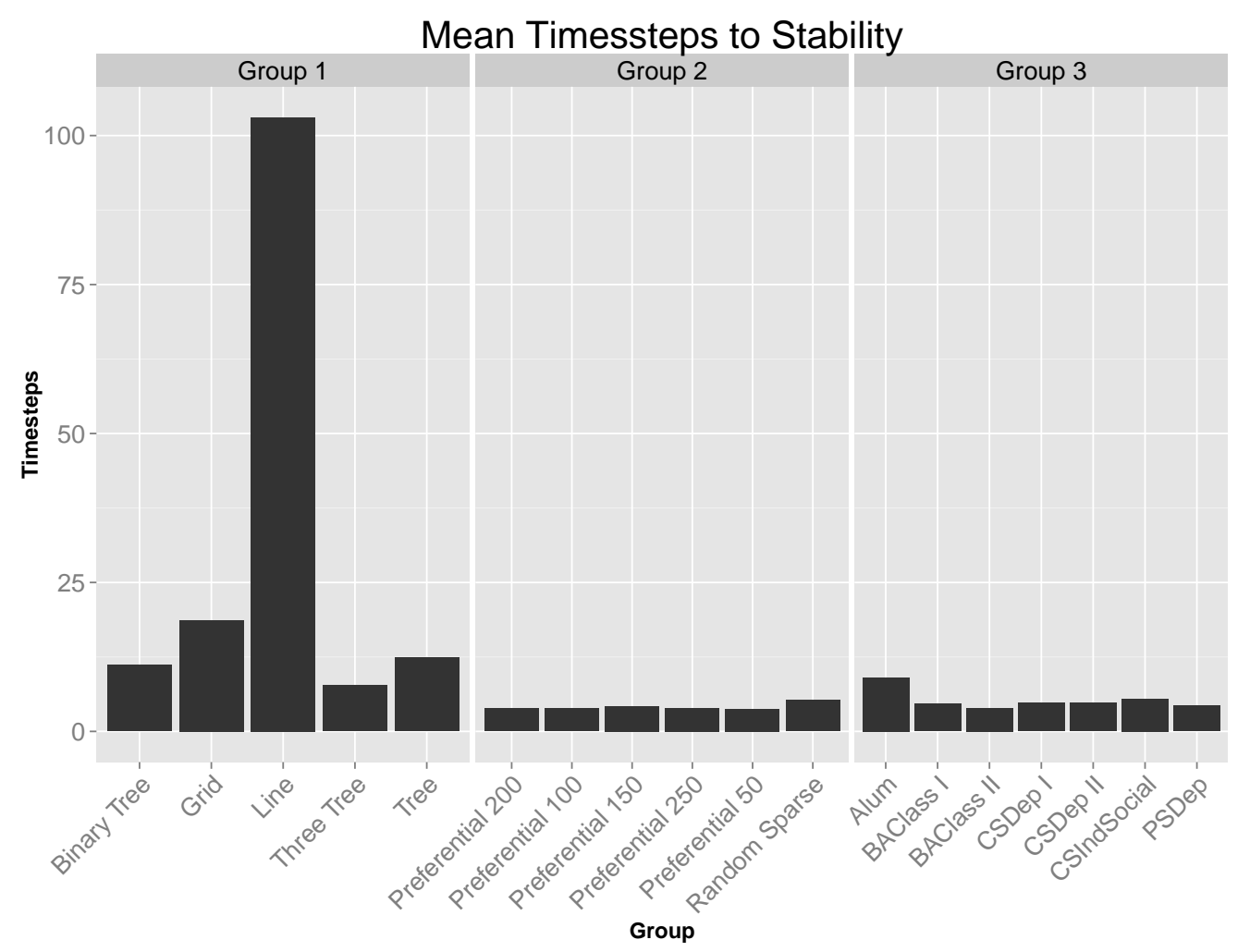

Figure 5.2: Graphs are listed on the $\mathrm{x}$-axis grouped by their type. The y-axis represents the average number of timesteps until stability for each network.

Note that the tree graphs and the line graph took about $d$ timesteps to become stable, where $d$ is the diameter of the graph. The first section of this chapter will prove that all tree and forest graphs will take $O(d)$ timesteps to come to a stable state. 


\section{$5.1 \quad$ Trees}

Definition 5.1.1. A node $v$, is balanced when $\left|B_{v}\right|=\left|S_{v}\right|$.

Lemma 5.1.1. Stability of number on trees will be achieved within $O(d)$ timesteps.

Proof. Let $v$ be a vertex on a tree $G$ such that $v \notin B \cup S$.

If $v$ is removed from $G$, the resulting graph $G^{\prime}$ will be disconnected, by the definition of a tree, and none of $v$ 's neighbors will be connected to each other by any path.

Because each opinion is seeded in the graph by one expert, then passed along paths to the rest of the nodes in the graph, only the nodes in the same connected component of $G^{\prime}$ as an initial expert can hold that expert's opinion.

Therefore if $y_{v}=\perp$, then $v$ cannot have two neighbors of the same opinion. This means a balanced node will never choose either opinion and so will not delay the stability of number.

Let $G^{\prime \prime}=G \backslash D$ where $D=\{v \mid v$ is balanced $\} \cup\{v \mid v \notin B \cup S$ and the only path from $\mathrm{v}$ to a node in $B \cup S$ goes through a balanced node\}.

In every timestep in which $|B| \cup|S| \neq\left|G^{\prime \prime}\right|$ there exist nodes $w$ such that $w \notin B \cup S$ but $w \in N_{B \cup S}$. By the definition of the model $w$ will be in $B \cup S$ in the next timestep.

Let $P(t)$ be the longest shortest path between any node not in $B(t) \cup S(t)$ and a node $w \in B(t) \cup S(t)$ such that $\exists u \in N_{w}, u \notin B(t) \cup S(t)$.

$|P|$ will be reduced by 1 in every timestep until $|P|=0$ because in every timestep $u$ will gain an opinion. Let $P(t)$ represent the shortest path between any node not in $B \cup S$ and the closest node in $B \cup S$. It will take at least $|P|(t)$ timesteps until $|B| \cup|S|=\left|G^{\prime \prime}\right|$. Because $P(t)$ also represents the longest path these points it will also take at most $|P(t)|$ timesteps.

Since the length of $P(t)$ can be at most the diameter, $d$, of the graph, stability of number will be reached in $O(d)$ steps.

Theorem 5.1.2. Stability of both number and opinion on trees will be achieved within $O(d)$ timesteps.

Proof. A leaf node, with only one neighbor will only be able to change opinion in the timestep after it initially gains an opinion because it is only in this timestep that 
there can be more neighbors of the opposing opinion. Therefore 2 timesteps after the leaf node gains an opinion it will be part of $F$, where $F$ is the set of nodes in $G$ that are stable.

For every graph $G$ for which $|G|>1$ there exists a node $v$ which has no more than one neighbor that is not a leaf. Every $v$ will become a part of $F$ at most one timestep after each of its children stabilize, four timesteps after it gains its initial opinion.

For our purposes, $v$ can now be considered a leaf node as it is stable and differing opinions can travel no farther down the tree.

Two timesteps after stability of number, while $|F| \neq|G|$, there will exist in every timestep at least one node with at most one neighbor not in $F$. Each of these nodes will join $F$ in the next timestep.

Let $P(t)$ be the longest shortest path between any node not in $F(t)$ and a node $w \in F(t)$ such that $w$ has a neighbor which is not in $F$.

$|P(t)|$ reduces by 1 in every timestep and $P(t)$ represents the shortest path between some node not in $F$ and the closest node in $F$, therefore it will take at least $|P(t)|$ timesteps for $|F|=|G| . P(t)$ also represents the longest path between any node not in $F$ and the closest node in $F$ therefore, it will take at most $|P(t)|$ timesteps for $|F|=|G|$

Since the length of $P(t)$ can be at most the diameter, $d$, of the graph, stability will be reached in $O(d)$ steps.

\subsection{A $\Omega\left(n^{\frac{3}{2}}\right)$ Lower Bound}

The Pure Opinion Model is the same as the Frischknecht, et al. Majority Rules Model in all but two minor points[22]. First, in the Majority Rules Model, the initial opinions of each vertex are randomly assigned instead of spreading from two experts. Second, nodes in Majority Rules Model never consider themselves part of their own neighborhood, where as in the Pure Opinion Model, the first timestep after gaining an opinion, nodes do not consider themselves part of their neighborhood, but in all future timesteps they do.

Although these differences do impact the time to stability, the following will prove the existence of networks which under the Pure Opinion Model have the same $\Omega\left(n^{\frac{3}{2}}\right)$ as found in Frischknecht et al.

Frischknecht et al. described a structure, shown in figure 5.3, called a transistor 
$T(k)$ of size $O(k)$, which is able to change the opinions of $\mathrm{O}\left(k^{2}\right)$ nodes in an adjoining path $P$. Initially all of the nodes in both the transistor and the path hold the same opinion. Then the transistor is triggered and all nodes in the transistor change opinion within 4 timesteps, after which the transistor changes the opinions of each node in the path one node at a time. Because the path is $O\left(k^{2}\right)$ in length the last node in the path will change its opinion $O\left(k^{2}\right)$ timesteps after the first node in the path.

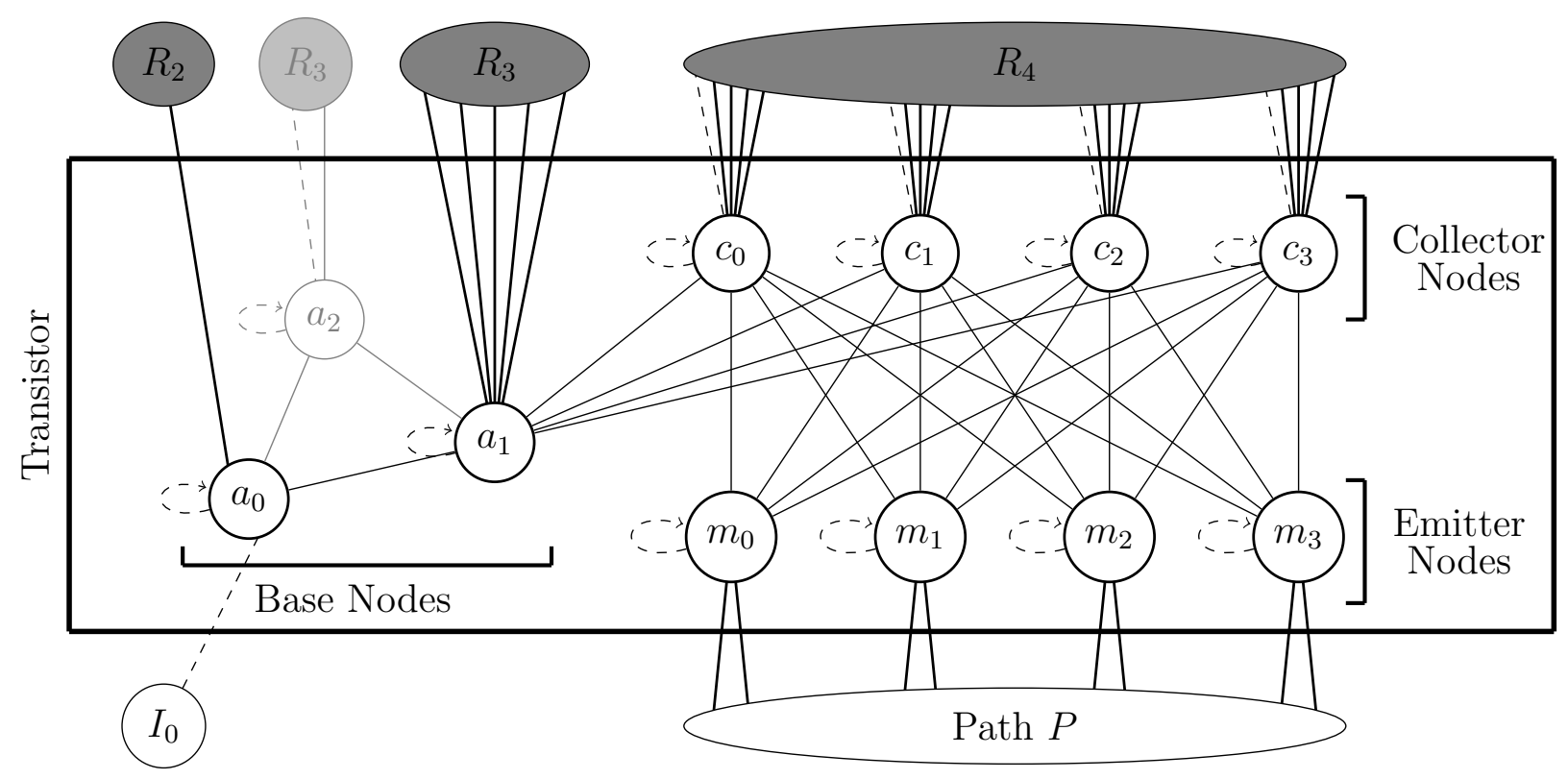

Figure 5.3: A transistor T(4). The solid lines represent edges that existed in the original Frischknecht et al. transistor, while dotted lines represent edges added to allow the system to work under the Pure Opinion Model. The nodes and paths that are light gray existed in the original transistor but have been removed from this one.

There are three subsets of nodes described in the Frischknecht et al.[22] transistor: the emitter nodes $M$, the collector nodes $C$, and the base nodes $a_{0}, a_{1} \in A^{1}$. Each of these sets of nodes must also exist for the transistor to work under the Pure Opinion model.

\footnotetext{
${ }^{1}$ As will be discussed later, there were 3 base nodes in the Majority Rules Model, however in the Pure Opinion Model only two will be used.
} 


$$
\begin{aligned}
T(k)= & (V, E) \\
V= & C \cup M \cup A \\
E= & \{\{u, v\} \mid u \in C, v \in M\} \\
& \cup\left\{\left\{u, a_{1}\right\} \mid u \in C, a_{1} \in A\right\} \\
& \cup\{\{u, v\} \mid u, v \in A\}
\end{aligned}
$$

In the Pure Opinion Model, without loss of generality, let the initial opinion of all nodes in the transistor $y_{T(k)}$ be 0 . Remember that $B(t)$ is the set of nodes holding the opinion 0 at time $t$ and $B_{v}(t)=\hat{N}_{v}$ such that $\forall w \in B_{v}(t), y_{w}(t)=0$. Similarly, $S(t)_{v}=\hat{N}_{v}$ such that $\forall w \in S_{v}, y_{w}(t)=1$.

The only nodes in the transistor which share edges with the nodes in the path are the $k$ emitter nodes. The other neighbors of any node $m \in M$ are the collector nodes, of which there are also $k$. It is the responsibility of the collector nodes to convert the nodes in $M$ when the transistor changes opinion; to ensure that this is always possible, a majority of every node $m \in M$ 's neighbors are nodes in $C$. The maximum number of neighbors $m$ has outside of $C$ (and to nodes in $P$ ) therefore is $k-2$. In the Majority Rules Model this number is $k-1$ as nodes in that model are not part of a closed neighborhood.

Each collector node has an edge with each of the $k$ emitter nodes as well as $k+1$ edges with supporter nodes $R$ which have the opposite opinion of the initial opinion in the transistor. That is, if the initial opinion of the transistor is 0 each collector node $c$ will have $\left|S(t)_{c}\right|=k+1$ because of the collector's neighbors in $R$, and $\left|B(t)_{c}\right|=k+1$ because of its neighbors in $M$ as well as $c$ itself. This balance is important as it allows the collector nodes to hold opinion 0 initially and then change to opinion 1 at the correct time.

Each collector node also has one more neighbor $a_{1}$, which is one of the base nodes in $A$. Initially $y_{a_{1}}=0$, as it is part of the transistor, when the transistor flips, the changing of $y_{a_{1}}$ to 1 is what tips the balance of the collector nodes neighborhoods from majority $B(t)$ opinion to majority $S(t)$, and in the next timestep changes their opinion.

Unlike the collector and emitter nodes, the number of base nodes does not change 
with the size of the transistor. There will always be two base nodes. Frischknecht et al. describe a transistor with three base nodes instead of two, their third base node was needed as a balance to the other two, something that is not needed in this model because nodes consider themselves part of their own neighborhood after the initial time step. In both models the base node, $a_{0}$ will be the first node to change its opinion when the transistor flips. $a_{0}$ is connected to: $a_{1}$, an initializer node which will seed the transistor with its initial opinion, a trigger node which will trigger the change in the transistor, and three support nodes.

As mentioned previously the second base node $a_{1}$ is responsible for triggering the change of the $k$ collector nodes. Before the transistor's change of opinion $\left|B_{a_{1}}(t)\right|=$ $k+2$, the $k$ neighbors it has in $C, a_{0}$, and itself, and $\left|S_{a_{1}}(t)\right|=k+1$, all of which are $a_{1}$ 's support nodes. When $a_{0}$ changes opinion one is taken away from $\left|B(t)_{a_{1}}\right|$ and one is added to $\left|S_{a_{1}}(t)\right|$, giving $\left|S_{a_{1}}(t+1)\right|$ a clear majority.

The transistor just described is very similar to the one used to gain the $\Omega\left(n^{\frac{3}{2}}\right)$ limit in Frischknecht et al. with the majority of the differences due to the fact that after the first timestep nodes in this paper consider their own opinion when deciding on their opinion. The other major difference, is that of how nodes gain their opinions in the first place, which still remains to be shown.

Lemma 5.2.1. If all nodes in transistor $T(k)$ as well as all but one of the support nodes have no opinion $\perp$ at time $t$ and $a_{0}$ has a neighbor $I$ with an opinion, then by $t+4$ it is possible to initialize every node in the transistor to the same opinion and every support node to the opposite opinion of that of the transistor.

Proof. Support nodes $R$ are nodes with the opposite opinion to that of the initial opinion of the transistor, which either have a neighbor in the transistor or neighbors in $R$ that have a neighbor in the transistor. Each subset of $R$ will be denoted with the timestep in which it initially gains an opinion as a subscript; additionally the subset of support nodes that do not have a neighbor in $T(k)$ will be denoted $R^{\prime}$. The transistor used by the Pure Opinion Model as well as all of its support nodes can be seen in figure 5.4 .

The initializer node $I$, gains an opinion and passes it along to every neighbor each of which pass the opinion along to all of their neighbors. One of the initializer's neighbors is $a_{0}$ and as long as none of $a_{0}$ 's other neighbors have adopted the opposing opinion in the previous timestep $a_{0}$ will adopt $I$ 's opinion. If $a_{0}$ is convinced of opinion 0 at time $i+1$, then at $i+2 a_{0}$ will pass its opinion on to $a_{1}$, which, similarly, will 
have no other neighbors with opinions. In the same timestep $a_{0}$ 's three support nodes $R_{2}$, will be convinced of opinion 1 by the two nodes which influence them which are in $R_{1}^{\prime}$. The nodes in $R_{1}^{\prime}$ will have gained their opinion from a node which in timestep $i$ held opinion $1, R_{0}^{\prime}$. The timing here is very important, had the nodes in $R_{2}$ gained the opposite opinion a timestep before $a_{0}$ or even in the same timestep they would have had a majority at $i+2$ and swayed $a_{0}$ to the opposite opinion.

Next, $a_{1}$ will adopt an opinion at $i+2$ and its support nodes, $R_{3}$ can adopt their opinion at $i+3$, influenced by $R_{2}^{\prime}$ which has two nodes. The collector nodes will also be initialized in $i+3$ and then at $i+4$, while their support nodes $R_{4}$ are being initialized by $R_{3}^{\prime}$, they will initialize the emitter nodes. To correctly balance the collector nodes there must be at least $k+1$ nodes in $R_{4}$ and to properly initialize those $R_{3}^{\prime}$ must have at least $k$ nodes.

The one node we have not discussed is the trigger node which is a neighbor of $a_{0}$ and $R_{1}^{\prime}$. At $i+2$ this node will have one neighbor with the 0 opinion and one with the 1 opinion and therefore be balanced and not have an opinion in $i+3$. The trigger node has a third neighbor, one which at some future timestep will gain opinion 1. When the trigger node holds the 1 opinion $a_{0}$ will change its opinion and within 4 timesteps the entire transistor will have flipped.

Once the transistor has changed opinions it will influence the nodes on path $P$ to change one at a time via the emitter nodes. The first node in $P$ to change its opinion will be $p_{0}$, the inner nodes of the path will be denoted $p_{i}{ }^{2}$ and $p_{P \mid-1}$ will be the last node on $P$.

Lemma 5.2.2. A transistor, $T(k)$ can change the opinion of all nodes on $P$, which is $O\left(k^{2}\right)$ in length, assuming $k>2$, in $\Omega\left(k^{2}\right)$ time.

Proof. A transistor has $k$ emitter nodes, $k$ collector nodes, two base nodes, $3 k+9$ support nodes, one initializer node, and a trigger node, for a total of $5 k+13$ nodes. For these $O(k)$ nodes to change the opinions of the $\mathrm{O}\left(k^{2}\right)$ nodes in $P$ in as many timesteps the nodes in $P$ must be converted to the new opinion one at a time, node $p_{0}$ will be the first node to have its opinion changed.

Again without loss of generality, when $t=0$ let $y_{P}(t)=0$. To change the opinion of a node, a majority of the node's neighbors must hold the opposite opinion. $\left|B_{p_{0}}(t)\right|=$

\footnotetext{
${ }^{2} \forall p_{i} \in P, N_{p_{i}} \cap P=2$
} 


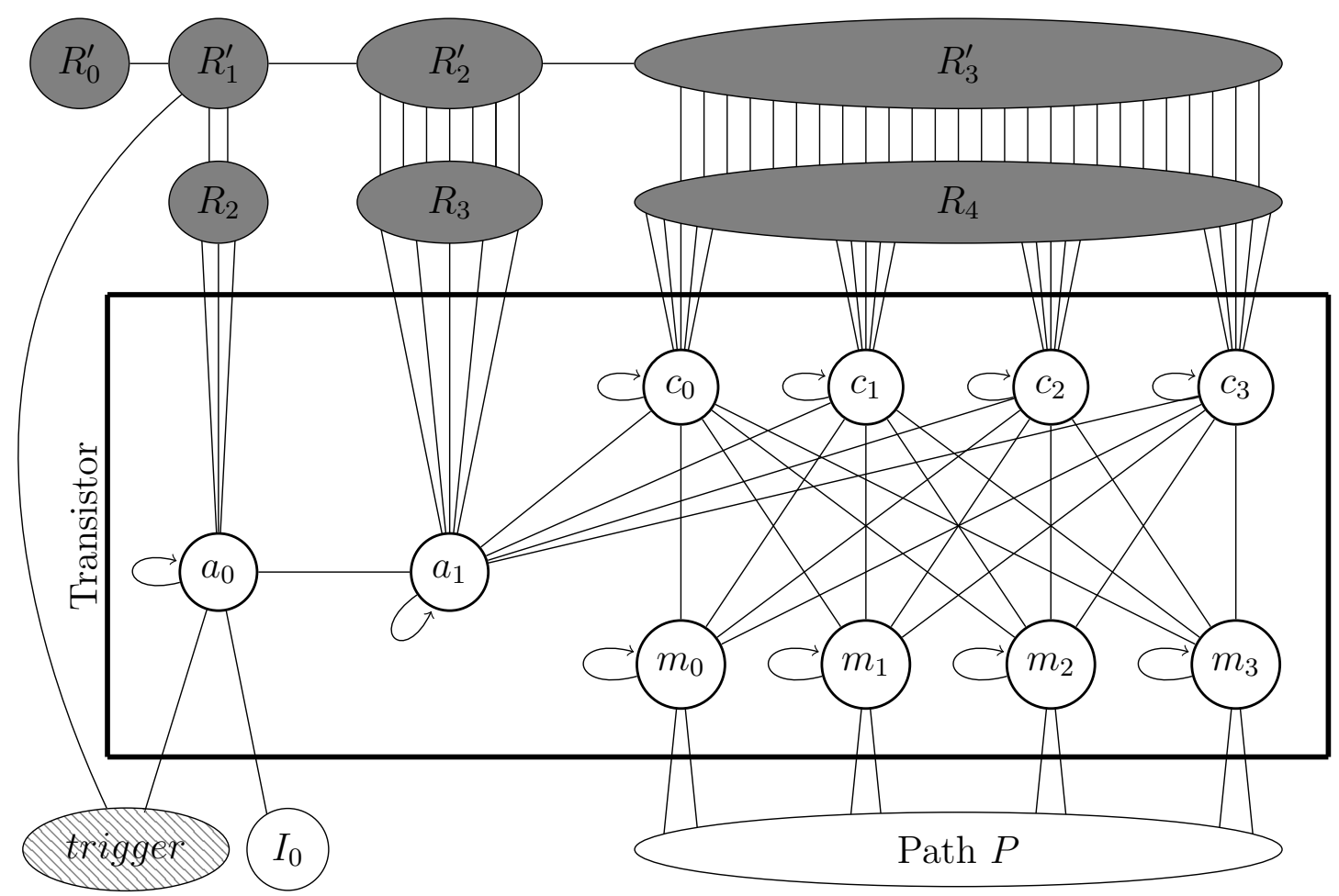

Figure 5.4: A transistor T(4). The white and gray nodes hold different opinions before the transistor is triggered and both hold the gray opinion after the transistor has been flipped. The trigger holds no opinion until it triggers the flip of the transistor.

2 , itself and the next node $p_{1} \in P$. In order to gain a majority therefore it must be true that $\left|S_{p_{0}}(t)\right| \geq 3$; each of these three nodes will be in the transistor.

Next, each inner node, $p_{i}$, must change its opinion only after the node before it, $p_{i-1}$, has changed opinion. Before the previous node has changed opinion $\left|B_{p_{i}}(t-1)\right|=$ 3: $p_{i-1}, p_{i}$, and $p_{i+1}$. When $p_{i-1}$ changes opinion $\left|B_{p_{i}}(t)\right|$ will decrease by one and $\left|S_{p_{0}}\right|$ will increase by one. For $y_{p_{i}}(t-1)$ to be correct $\left|S_{p_{i}}(t-1)\right|$ cannot have been greater than 3 and $\left|S_{p_{i}}(t)\right|$ cannot be less than 3 . Therefore $p_{i}$ can have two to three neighbors from the transistor if it is to change opinion at the appropriate time.

Finally, the last node in the path $p_{P \mid-1}$, must only change opinion once every other node in $P$ has changed opinion. $p_{|P|-1}$ has only one other neighbor in $P$, before that neighbor changes $\left|B_{p_{|P|-1}}(t)\right|=2$ after $\left|B_{p_{|P|-1}}(t)\right|=1$, meaning $\left|N_{p_{P \mid-1}} \cap T\right|$ can be 1 or 2 .

With at least three emitters connected to $p_{0}$, two connected to $p_{i}$, and one a neighbor of $p_{P \mid-1}$ the mean number of edges per node in $P$ is two. There are $k$ emitter nodes, each of which can have up to $k-2$ neighbors not in $T$. The length of 
$P$ therefore can be $\frac{k(k-2)}{2}$, which is $O\left(k^{2}\right)$, and since nodes on the path change their opinion one at a time this path will change in $\Omega\left(k^{2}\right)$.

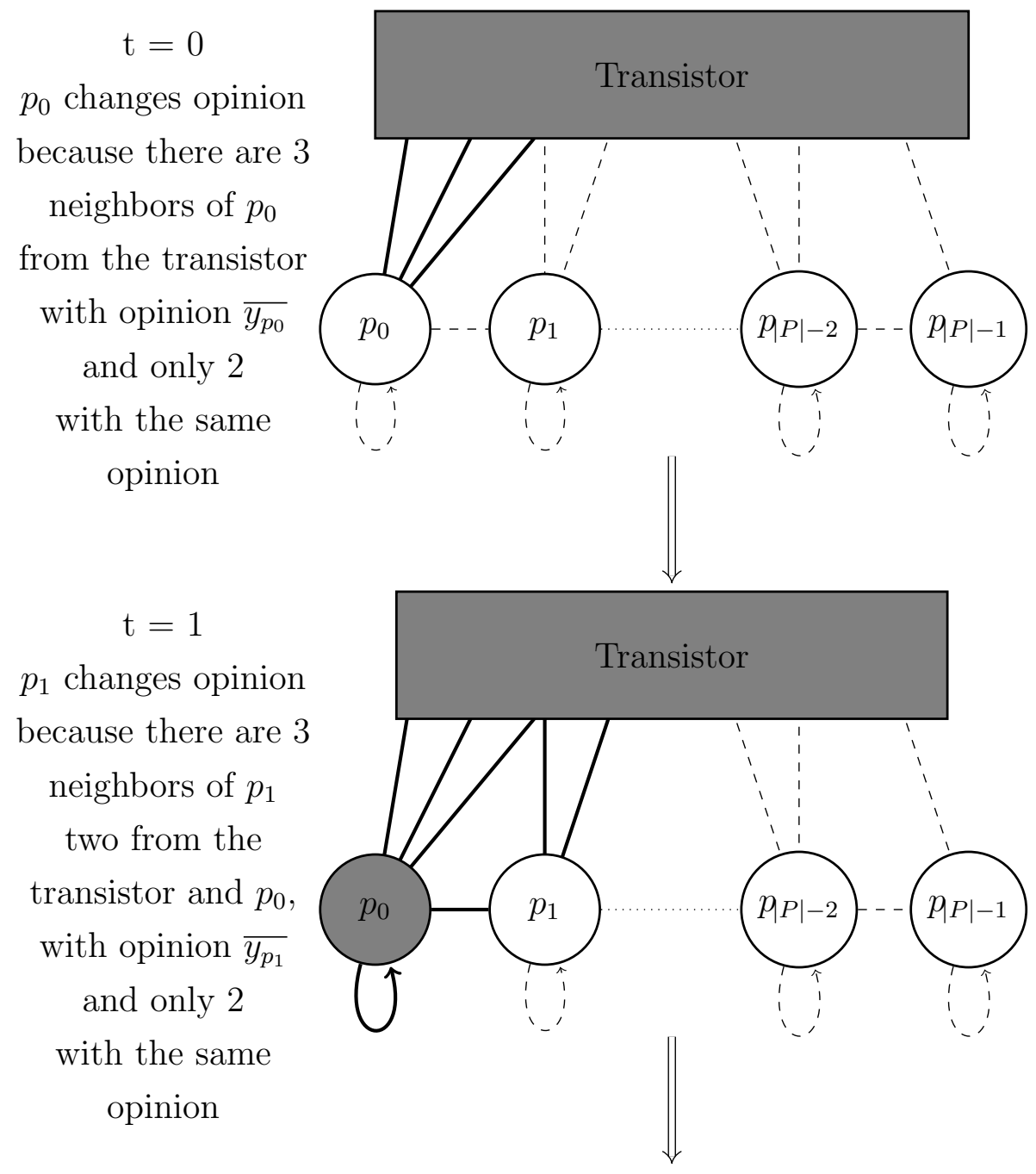




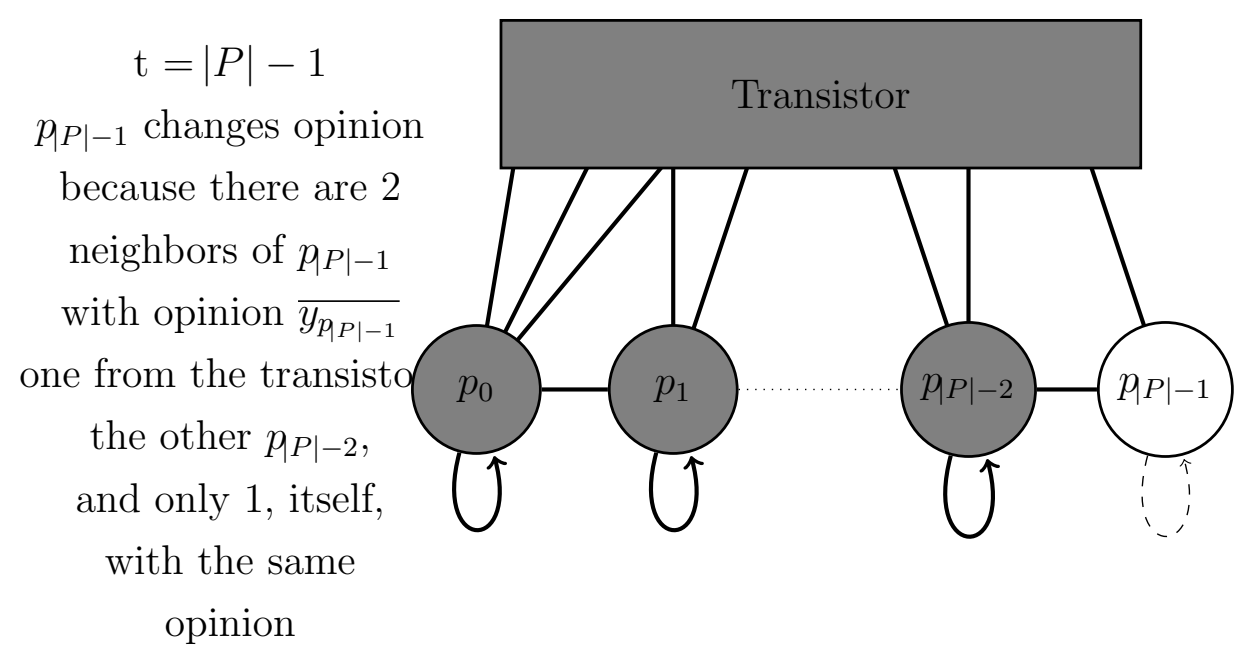

Figure 5.4: The nodes of the path initially have an opinion that is represented by white and are influenced by the transistor to change to the opinion represented by gray. The solid lines show edges that influence the node to be gray. Dashed lines influence a node to be white.

When there is more than one transistor connected to a path then in order to trigger the second transistor properly there must be a second mirrored path holding and being convinced of the opposite opinion at every timestep.
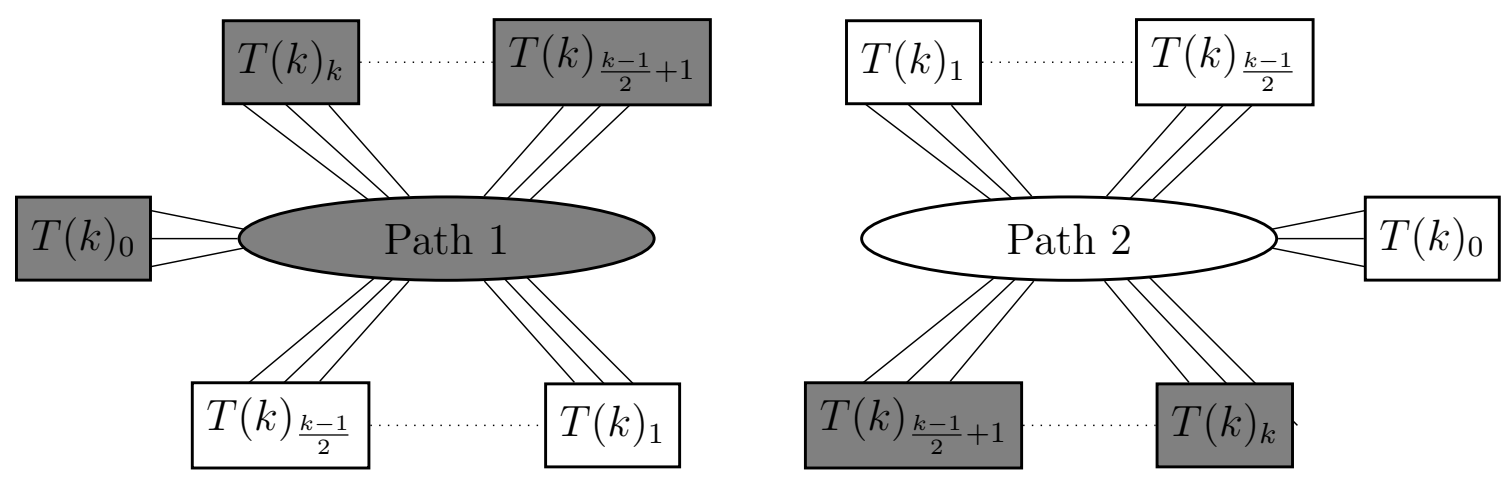

Figure 5.5: Two mirrored paths, each with $k$ transistors.

Each path is connected to an odd number of transistors and at every timestep there is exactly one more transistor of one opinion than the other connected to each path. If that opinion does is not the same opinion as the path then the path will change opinion. 


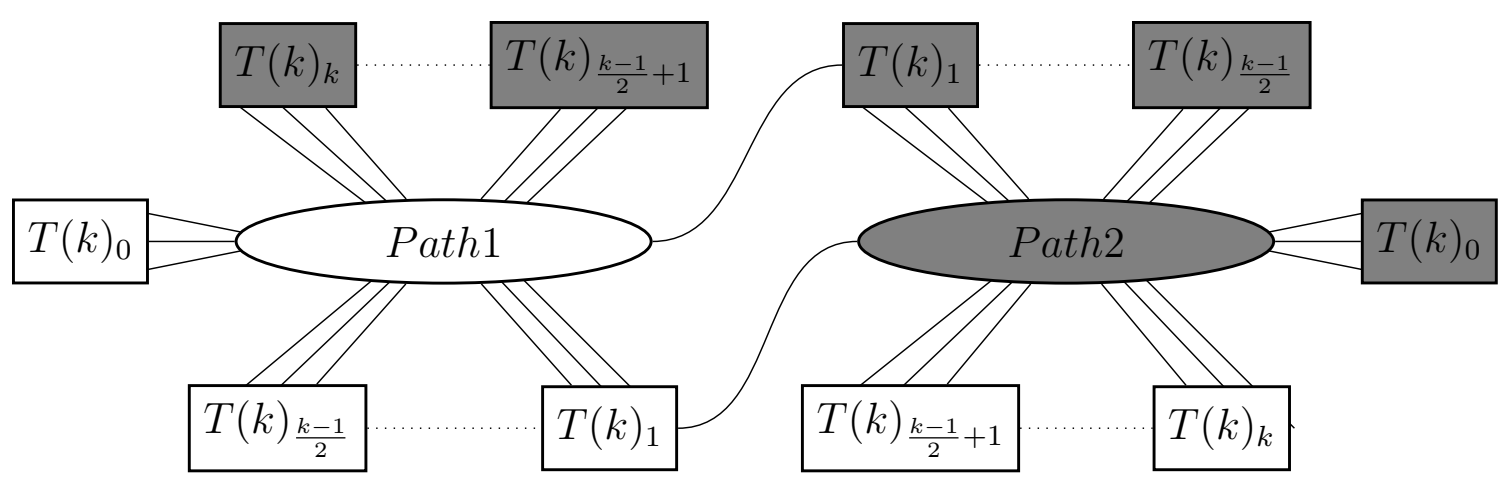

The last node on each path then triggers the change of the next transistor for the other path. When those transistors are flipped they change the opinions of the paths they belong to. Transistors can only ever change opinion once as after they change the balance of their support nodes is broken, this is why $k$ transistors are needed to flip a path $k$ times instead of only two transistors of opposing opinions.

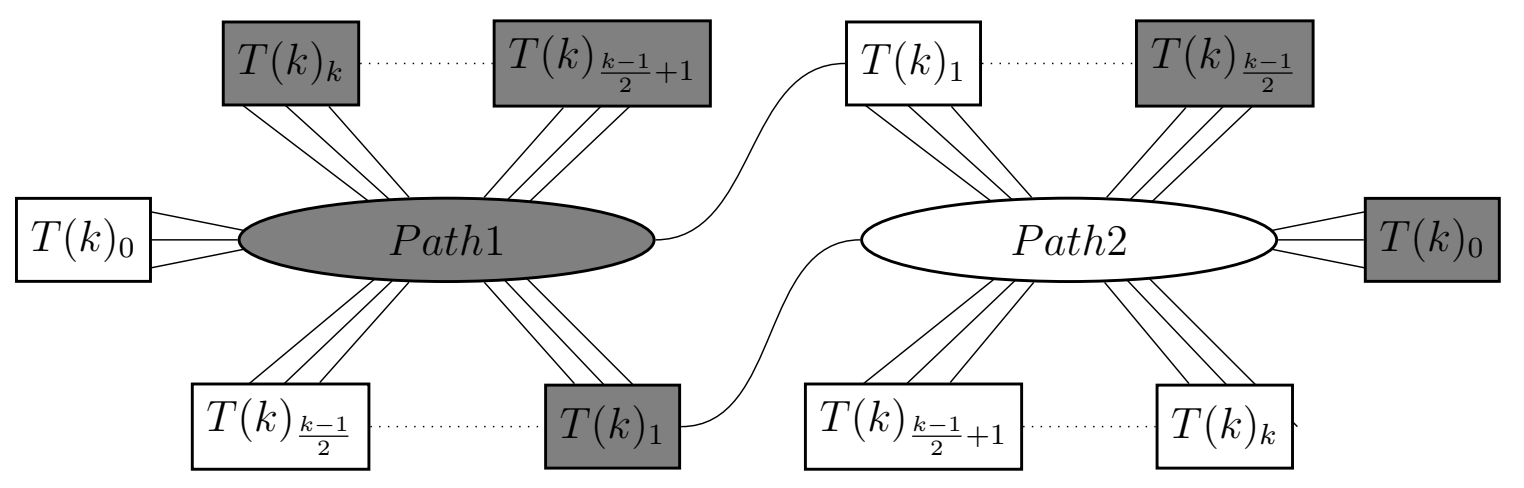

The newly flipped path now triggers the next transistor $T(k)_{i} . T(k)_{i}$ was not changed last time the path held this opinion because it must be triggered not only by the path but by the transisor $T(k)_{i-1}$ that most recently flipped that path, and the last time the path held its current opinion the $T(k)_{i-1}$ had not yet changed opinion.

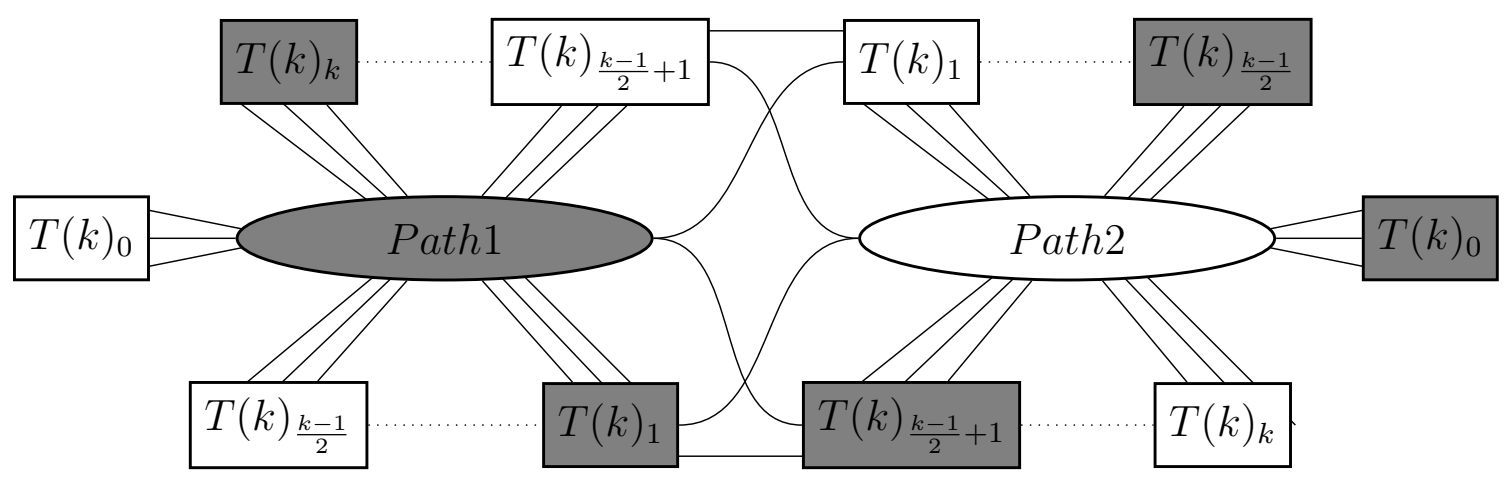


For every subsequent transistor change both the last node on the path and the previous transistor will be needed to change the opinion of the path.

Lemma 5.2.3. There exists a $k$ for which $k$ transistors of size $k$ can be initialized to change the opinion of all nodes on a path of length $O\left(k^{2}\right), k$ times.

Proof. Let $p_{0}$ be the starting node of the path.

As long as there are an odd number of transistors connected to a path in the same way as the transistor was described connected in Lemma 5.2.2 the conversion of a path will happen just as before. Let the number of transistors connected to a path be NT. Other than the most recently flipped transistor exactly half the transistors, $\frac{N T-1}{2}$ will be of one opinion, the other half of the other opinion, at all times. When a transistor flips it will create a majority, if it changes to opinion 1 then in the next timestep $\left|B(t)_{p_{0}}\right|=3\left\lfloor\frac{N T}{2}\right\rfloor+2$ and $\left|S(t)_{p_{0}}\right|=3\left\lfloor\frac{N T}{2}\right\rfloor+3$.

Similarly $p_{i}$ will change when $\left|B(t)_{p_{i}}\right|=2\left\lfloor\frac{N T}{2}\right\rfloor+2$ and $\left|S(t)_{p_{0}}\right|=2\left\lfloor\frac{N T}{2}\right\rfloor+3$, and $p_{P \mid-1}$ will flip when $\left|B(t)_{p_{P \mid-1}}\right|=\left\lfloor\frac{N T}{2}\right\rfloor+1$, and $\left|S(t)_{p_{|P|-1} \mid}\right|=\left\lfloor\frac{N T}{2}\right\rfloor+2$.

Initializing these additional transistors is a simple task. The initializer nodes of every transistor, with the exception of two, will be connected through a path 2 in length to the starting node of their initial opinion. This starting node will also be connected by a path of 2 to the $R_{0}^{\prime}$ node for each transistor initialized to the opposite opinion. The exception of two transistors will be the transistors to initialize the paths, these transistors will be connected to their respective starting nodes by a path of only one. The initialization of the path will not effect the opinion of the other transistors as their emitter nodes will be initialized in the same timestep as the path and the emitter nodes cannot be swayed by their neighbors outside of $T(k)$.

Finally each transistor must be triggered to flip at the proper time. The first two transistors will be triggered simultaneously, adopt opposite opinions, and connected to different paths. This may happen through a path of nodes leading from their $R_{4}$ support nodes or some other node of opposing opinion. Once triggered the transistors will convince the respective paths of their new opinions. Each $p_{P \mid-1}$ will then trigger the next transistor for the other path. As long as each $p_{P \mid-1}$ is connected to a balanced number of transistors, at each timestep in which another transistor is trying to convert it this will not effect how $p_{P \mid-1}$ is flipped.

This path change as the sole trigger will only work with the first transistor triggered by the end of the path. In order to avoid half of the transistors flipping in the timestep after the last node in the path changes opinion, subsequent transistors 
will be triggered only after both a path and the most recent transistor to change the opinion of that path adopt their new opinion.

For example, as can be seen in Figure 5.6, one path prompts a transistor, $T(k)_{1}$ to change. $T(k)_{1}$ is connected to the trigger of the next transistor $T(k)_{2}$ but the trigger still has the agreement of the path it is connected to as well as $T(k)_{2}$ and is not swayed to the new opinion. $T(k)_{1}$ then convinces the path of the new opinion and together with another balancing node they convince the trigger of the new opinion. The path was unable to convince the trigger before $T(k)_{1}$ changed opinion as it would not yet have had a majority.

Unlike the trigger described in Lemma 5.2.2, triggers which change opinion only after a path or a path and a transistor have changed opinion will not be balanced with no opinion but rather will match the opinion of the transistor they are connected to until flipped.

This additional edge from each transistor to the next transistor to be flipped means that the length of the path changes from $\left\lfloor\frac{k(k-2)}{2}\right\rfloor$ to $\left\lfloor\frac{k(k-2)-1}{2}\right\rfloor$. This does not change the time complexity however and stability will be reached on these paths after $\Omega\left(k^{3}\right)$ timesteps.

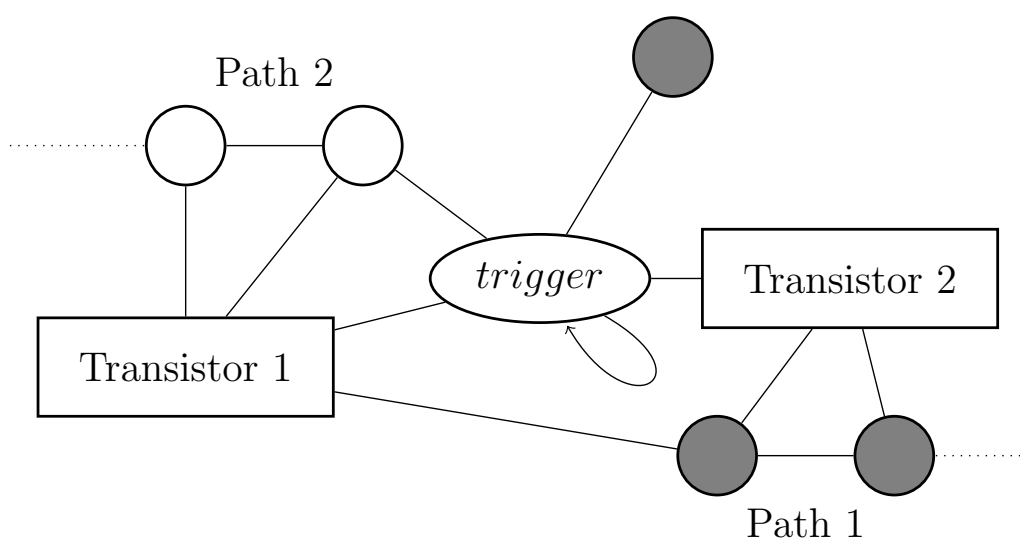

Figure 5.6: The trigger node will not flip Transistor 2 until both Transistor 1 and Path 2 have changed to the gray opinion. If Path 2 is gray in previous timesteps Transistor 1 will not have switched and trigger will have 3 to 2 in favor of staying white. When Transistor 1 does flip we know that Path 2 will be white so trigger will maintain the ratio in favor of remaining white. Only when Transistor 1 convinces Path 2 of gray will the number of trigger's neighbors favoring gray be high enough to change its opinion and subsequently trigger the flip of Transistor 2. 
Theorem 5.2.4. A graph with the same lower bound time complexity of $\Omega\left(n^{\frac{3}{2}}\right)$ as found in Frischknecht et al., exists for the Pure Opinion Model.

Proof. Lemma 5.2.2 shows that $O(k)$ nodes can change $O\left(k^{2}\right)$ nodes in $\Omega\left(k^{2}\right)$ timesteps. Lemma 5.2.3 proved that this process could be repeated $k$ times meaning the graph would stabilize in $\Omega\left(k^{3}\right)$ timesteps. There are $5 k+13$ nodes in each transistor and two paths of $O\left(k^{2}\right)$ nodes meaning the total $n$ is $O\left(k^{2}\right)$, therefore in terms of $n$ the graph will take $\Omega\left(n^{\frac{3}{2}}\right)$ to stabilize. 


\section{Chapter 6}

\section{Future Work}

The Certainty Model reaches stability $O(d)$ timesteps after reaching stability of number which is reached in $O(n)$. One of the main reasons for the $O(d)$ limit is that the most certain nodes' certainty could never increase even if every one of their neighbors agreed with them. In future research it would be interesting to explore different ways nodes could increase their certainty. For example, for every timestep in which all of a node's neighbors agreed with it, a node might increase in certainty a certain amount. This amount could vary depending on the certainty or number of the node's neighbors, or it could just be a fixed number.

Another way to change the way in which certainty is calculated might be a model in which nodes do not take each of their neighbors' opinions into consideration at every timestep. Rather if a node was contacted by some neighbor who disagreed with it, the node would lose certainty and reach out to another neighbor in the next timestep. If the second neighbor agreed with the node's original opinion its certainty would be restored. If the second neighbor did not hold the same opinion the node's certainty would decrease again, and again it would seek reassurance from yet another neighbor. After a certain threshold of lost certainty, the node would convert to the opposing opinion. In the average case this model would probably stabilize fairly quickly, however it would be interesting to find an upper bound of time to stability, if stability can be guaranteed to exist at all.

Alternatively certainty could be maintained by adding varying numbers of stubborn and conforming nodes as previously studied by Ghaderi et al. and Das et al. $[25,10]$. The difference is that previous studies have not dealt with competing opinions but rather a spectrum and previous studies have not considered the weighting of neighbors' opinions by certainty. 
Something both the Pure Opinion Model and the Certainty Model have in common is that in the initial timestep of both, there exist only two experts of opposing opinion. It would be interesting to explore how this initial set-up affects other models, such as those studied by Ghaderi, Axelrod, Deffuant et al., and DeGroot, in which initial opinions are often randomly assigned [25, 3, 13, 14]. Alternatively how much would the models change if there were more than two experts? If the experts still only represented two opinions, the upper stability bound $O(n)$ would probably remain unchanged and once stability of number was established not much else would be affected from this stability having been established slightly more quickly in the average case. However if there were more than two opinions represented in the initial set of experts, or a third opinion could appear at some later timestep, new patterns might be observed.

The current idea of a node gaining confidence in its own opinion could be expanded upon in both models, as well. For example, every $x$ number of timesteps that a node holds a certain opinion, its self confidence could increase. There could also be varying degrees of "open mindedness" so some nodes would be more easily swayed while others would more quickly gain a high degree of self confidence. I do not doubt that under this system the path to stability, and even the meaning of stability, would change a great deal.

The Pure Opinion Model on trees stabilizes in $O(d)$ time and on specific graphs with transistors stabilizes within $\Omega\left(n^{\frac{3}{2}}\right)$ timesteps. However it would be interesting to prove a time bound for sparse connected networks that are not necessarily trees. Another thing to explore is the time bound for reaching periodic stability.

Finally, an expansion of this research with practical marketing applications would be an investigation of which opinion is most represented when stability is reached. This research would probably look at the role of the structure of the graph in determining the time to stability and expand on the findings of section 5.1 where the $O(d)$ timesteps limit on a tree graph was found. 


\section{Bibliography}

[1] Jeff Alstott, Stuart Madnick, and Chander Velu. Homophily and the speed of social mobilization: The effect of acquired and ascribed traits. PLoS ONE, 9(4), 2014.

[2] Solomon E Asch. Studies of Independence and Conformity: 1.A Minority of One Against a Unanimous Majority. Psychological Monographs, 70(9), 1956.

[3] Robert Axelrod. The Dissemination of Culture. The Journal of Conflict Resolution, 41(2):203-226, 1997.

[4] Sven Banisch, Tanya Araújo, and Jorge Louçã. Opinion Dynamics and Communication Networks. Advances in Complex Systems, 13(01):95-111, 2010.

[5] Arnab Bhattacharyya, Mark Braverman, Bernard Chazelle, and Huy L. Nguyen. On the Convergence of the Hegselmann-Krause System. 2013.

[6] Robert M. Bond, Christopher J. Fariss, Jason J. Jones, Adam D. I. Kramer, Cameron Marlow, Jaime E. Settle, and James H. Fowler. A 61-million-person experiment in social influence and political mobilization. Nature, 489(7415):295298, 2012.

[7] Rod Bond and Peter B Smith. Culture and conformity: a meta-analysis of studies using Asch's (1952b 1956) line judgement task. Psychological bulletin, 119(1):111-137, 1996.

[8] D. Centola. An Experimental Study of Homophily in the Adoption of Health Behavior. Science, 334(6060):1269-1272, 2011.

[9] J. H. Christakis, N. A., \& Fowler. The collective dynamics of smoking in a large social network. New England journal of medicine, 358(21):2249-2258, 2008. 
[10] Abhimanyu Das, Sreenivas Gollapudi, Arindam Khan, and Renato Paes Leme. Role of Conformity in Opinion Dynamics in Social Networks. Proc. Intl. Conference on Social Networks (COSN), page 13, 2014.

[11] Abhimanyu Das, Sreenivas Gollapudi, and Kamesh Munagala. Modeling Opinion Dynamics in Social Networks. page 10, 2014.

[12] Abir De, Sourangshu Bhattacharya, Parantapa Bhattacharya, Niloy Ganguly, and Soumen Chakrabarti. Learning a Linear Influence Model from Transient Opinion Dynamics. In Proceedings of the 23rd ACM International Conference on Conference on Information and Knowledge Management - CIKM '14, pages 401-410. ACM, Shanghai, China, 2014.

[13] Guillaume Deffuant, David Neau, Frederic Amblard, and Gérard Weisbuch. Mixing beliefs among interacting agents. Advances in Complex Systems, 03(01n04):87-98, 2000 .

[14] Morris H. DeGroot. Reaching a Consensus. Journal of American Statistical Association, 69:118-121, 1974.

[15] David Easley and Jon Kleinberg. Cascading Behavior in Networks. In Networks, Crowds, and Markets: Reasoning about a Highly Connected World, chapter 19, pages 563-609. Cambridge University Press, 2010.

[16] David Easley and Jon Kleinberg. Chapter 16 Information Cascades. In Networks, Crowds, and Markets: Reasoning about a Highly Connected World., chapter Chapter 16, pages 483-508. Cambridge University Press, 2010.

[17] Glenn Ellison. Learning, Local Interaction, and Coordination. The Econometric Society, 61(5):1047-1071, 1993.

[18] S. Eubank, V.S.a. Kumar, M.V. Marathe, a. Srinivasan, and N. Wang. Structure of social contact networks and their impact on epidemics. DIMACS Series in Discrete Mathematics and Theoretical Computer Science, 70(0208005):181, 2006.

[19] Santo Fortunato. Damage spreading and opinion dynamics on scale-free networks. Physica A: Statistical Mechanics and its Applications, 348:683-690, 2005.

[20] John R French. A formal theory of social power. Psychological review, 63(3):181194, 1956. 
[21] Noah E. Friedkin. A formal theory of social power. The Journal of Mathematical Sociology, 12(2):103-126, 1986.

[22] Silvio Frischknecht, Barbara Keller, and Roger Wattenhofer. Convergence in ( Social ) Influence Networks. In Distributer computing, pages 433-446. 2013.

[23] Serge Galam. The dynamics of minority opinions in democratic debate. Physica A: Statistical Mechanics and its Applications, 336(1-2):56-62, 2004.

[24] F. Gargiulo and S. Huet. Opinion dynamics in a group-based society. EPL (Europhysics Letters), 91(5):5, 2010.

[25] J Ghaderi and R Srikant. Opinion dynamics in social networks: A local interaction game with stubborn agents. American Control Conference (ACC), 2013, pages 1982-1987, 2012.

[26] Rainer Hegselmann and Ulrich Krause. Opinion Dynamics and Bounded Confidence: Models, Analysis and Simulation. Journal of Artificial Societies and Social Simulation, 5(3), 2002.

[27] Rainer Hegselmann and Ulrich Krause. Opinion dynamics driven by various ways of averaging. Computational Economics, 25(4):381-405, 2005.

[28] Peng Jia, Anahita Mirtabatabaei, Noah E. Friedkin, and Francesco Bullo. Opinion Dynamics and the Evolution of Social Power in Influence Networks. (March):1-27, 2013.

[29] Luo-luo Jiang, Da-yin Hua, and Ting Chen. Nonequilibrium phase transitions in a model with social influence of inflexible units. Journal of Physics A: Mathematical and Theoretical, 40(37):11271-11276, 2007.

[30] Michihiro Kandori, George J Mailath, and Rafael Rob. Learning, Mutation, and Long Run Equilibria in Games. Econometrica, 61(1):29-56, 1993.

[31] David Kempe, Jon Kleinberg, and Éva Tardos. Maximizing the spread of influence through a social network. Proceedings of the ninth ACM SIGKDD international conference on Knowledge discovery and data mining - KDD '03, page 10, 2003. 
[32] Christopher B Kenny and B Kenny. Political Participation and Effects from the Social Environment *. 36(1):259-267, 2012.

[33] M. F. Laguna, S. Risau Gusman, G. Abramson, S. Gonçalves, and J. R. Iglesias. The dynamics of opinion in hierarchical organizations. Physica A: Statistical Mechanics and its Applications, 351(2-4):580-592, 2005.

[34] Raglan Maddox, Rachel Davey, Ray Lovett, Anke van der Sterren, Joan Corbett, and Tom Cochrane. A systematic review protocol: social network analysis of tobacco use. Systematic reviews, 3(1):85, 2014.

[35] Anahita Mirtabatabaei and Francesco Bullo. On opinion dynamics in heterogeneous networks. Proceedings of the 2011 American Control Conference, pages 2807-2812, 2011.

[36] Andrea Montanari and Amin Saberi. Convergence to equilibrium in local interaction games. Proceedings - Annual IEEE Symposium on Foundations of Computer Science, FOCS, 8(1):303-312, 2009.

[37] David W. Nickerson. Is Voting Contagious? Evidence from Two Field Experiments. American Political Science Review, 102(01):49-57, 2008.

[38] Walter Quattrociocchi, Guido Caldarelli, and Antonio Scala. Opinion dynamics on interacting networks: media competition and social influence. Scientific reports, 4:4938, 2014.

[39] M. Rosvall and K. Sneppen. Dynamics of Opinions and Social Structures. page 4, 2008.

[40] David O. Sears. Social anxiety, opinion structure, and opinion change. Journal of Personality and Social Psychology, 7(2, Pt.1):142-151, 1967.

[41] Michael B Walker and Maria G Andrade. Conformity in the Asch Task as a Function of Age. Journal of Social Psychology, 136(3):367-372, 1996.

[42] Haoxiang Xia and Huili Wang. Opinion Dynamics: Disciplinary Origins, Recent Developments, and a View on Future Trends. Iskss.Org, 8:1-6, 2001.

[43] Ercan Yildiz, Daron Acemoglu, Asuman Ozdaglar, Amin Saberi, and Anna Scaglione. Binary Opinion Dynamics with Stubborn Agents. ACM Transactions on Economics and Computation, 1(4):30, 2013. 
[44] Ercan Yildiz, Asu Ozdaglar, Daron Acemoglu, and Anna Scaglione. The voter model with stubborn agents extended abstract. 2010 48th Annual Allerton Conference on Communication, Control, and Computing, Allerton 2010, pages 11791181, 2010.

[45] Damián H. Zanette and Santiago Gil. Opinion spreading and agent segregation on evolving networks. Physica D: Nonlinear Phenomena, 224(1-2):156-165, 2006.

[46] Damián H. Zanette and Sebastián Risau-Gusmán. Infection spreading in a population with evolving contacts. Journal of Biological Physics, 34(1-2 SPEC. ISS.):135-148, 2008.

[47] Jiangbo Zhang and Yiguang Hong. Convergence analysis of heterogeneous Deffuant-Weisbuch model. Control Conference (CCC), 2012 31st Chinese, pages 1124-1129, 2012. 